\title{
Correction dynamique des distorsions de faisceaux laser par interactions non linéaires
}

\author{
A. Brignon \\ Laboratoire Central de Recherches, Thomson-CSF, Domaine de Corbeville, \\ 91404 Orsay cedex, France
}

\begin{abstract}
Résumé: Les lasers solides présentent des avantages relatifs à leur compacité, facilité d'utilisation et à leur durée de vie. Cependant, une partie importante de l'énergie de pompage se dissipe sous forme de chaleur provocant des distorsions de phase importantes dans le milieu laser. Cet effet indésirable dégrade la qualité spatiale du faisceau émis et peut abaisser considérablement la luminance du laser. Les techniques de conjugaison de phase par interactions non-linéaires permettent de corriger ces distorsions de façon adaptative et dynamique. Après avoir examiné le principe de la conjugaison de phase, nous passons en revue les principales interactions nonlinéaires permettant d'obtenir cette fonction. Nous étudions en particulier la diffusion Brillouin stimulée, l'effet photoréfractif et les mélanges d'ondes clans le milieu laser lui-même.
\end{abstract}

\section{INTRODUCTION}

Pour de nombreuses applications, il est indispensable de réaliser des sources lasers à haute énergie ou à puissance moyenne élevée conservant toutefois une très bonne qualité de faisceau (limité par la diffraction). De telles sources utilisent généralement des amplificateurs solides. S'ils présentent des avantages relatifs à leur compacité, facilité d'utilisation et à leur durée de vie, ils dégradent cependant les qualités spatiales de la lumière. En effet, la chaleur dissipée lors du pompage du milieu laser induit un gradient thermique lans ce dernier. Ce gradient thermique induit à son tour un gradient d'indice qui distord spatialement la phase de l'onde amplifiée.

Les sources lasers qui fonctionnent à fortes puissances moyennes doivent donc inclure des éléments optiques pour corriger ces distorsions. Pour cela, des lentilles ou des miroirs déformables peuvent être utilisés. Mais ces méthodes sont adaptées à des déformations constantes ou ne variant que faiblement dans le temps. Le gradient de température dépendant des conditions d'utilisation (fréquence de répétition, énergie de pompage ...), les déformations induites sur le faisceau en dépendent donc également. Or, pour de nombreuses applications, il est souhaitable de conserver des faisceaux de grande qualité de front d'onde quel que soit le régime d'utilisation. De plus, les distorsions induites ne sont pas toujours parfaitement sphériques. Toutes ces contraintes imposent donc une correction dynamique dans le temps, mais également adaptative spatialement. Une méthode particulièrement adaptée consiste à utiliser des miroirs non-linéaires à conjugaison de phase. De tels miroirs renvoient une onde dont la phase en tout point est une copie inversée de celle 
de l'onde incidente. Ainsi, l'association d'un amplificateur et d'un miroir à conjugaison de phase permet d'amplifier une onde laser sans en modifier la divergence et ceci quelles que soient les distorsions de phase introduites par l'amplificateur.

Cette fonction de conjugaison de phase peut être obtenue par des interactions d'ondes optiques dans les matériaux non-linéaires dont, d'une façon générale, l'indice, l'absorption ou le gain changent avec l'intensité lumineuse [1]. Ces propriétés non-linéaires se rencontrent dans des matériaux possédant une susceptibilité du troisième ordre, $\chi^{(3)}$, ou d'ordre supérieur non nul. C'est le cas notamment des miroirs non-linéaires utilisant la diffusion Brillouin stimulée. Ce type de miroir s'est déjà montré très efficace pour compenser les distorsions d'amplificateurs lasers de puissance fonctionnant à la limite de diffraction avec une très grande stabilité de pointé du faisceau. Ces miroirs non-linéaires connus depuis plus d'une vingtaine d'années, font maintenant partie intégrante de lasers commerciaux. Les miroirs Brillouins efficaces utilisent en général des milieux liquides ou gazeux, ce qui peut être gênant pour certaines applications.

Parmi les matériaux solides envisageables, les cristaux photoréfractifs constituent une solution intéressante. Les miroirs à conjugaison de phase réalisés avec ces cristaux permettent de remplacer les cellules Brillouin de façon avantageuse, notamment en terme de compacité et de facilité d'utilisation. Par ailleurs, dans les sources lasers où un miroir à conjugaison de phase ne peut être utilisé (régime continu, milieux lasers à faible gain, ...), le "nettoyage de faisceau" par mélange à deux ondes dans un cristal photoréfractif est une alternative intéressante pour améliorer la qualité spatiale du faisceau.

Une autre idée consiste à utiliser le milieu laser lui-même comme milieu non-linéaire. Le mécanisme physique mis en jeu est la saturation du gain laser. Lorsque plusieurs ondes interfèrent dans le milieu laser, le gain du milieu au niveau des zones claires des franges d'interférence sera différent de celui situé dans les zones sombres, créant ainsi cles réseaux de gain (ou réseaux d'amplitude). En utilisant; par exemple, une interaction dite de mélange à quatre ondes, la diffraction des ondes sur ces réseaux peut engendrer un faisceau conjugué en phase. Cette technique présente de très nombreux avantages et permet notamment la réalisation de sources lasers tout à l'état solide autocorrigées de leurs distorsions de phase.

\section{DISTORSIONS SPATIALES INDUITES DANS UN MILIEU LASER SOLIDE}

Les distorsions d'un milieu amplificateur solide proviennent principalement d'effets thermiques induits lors du pompage optique par lampes flash ou diodes lasers par exemple [2-4]. En effet, l'énergie de la pompe n'est que partiellement transférée au niveau haut de la trausition laser. Une fraction, qui peut être très importante, de la puissance fournie par la pompe est alors transformée en chaleux. Une partie de cette chaleur peut être partiellement évacuée en périphérie du milieu par un circuit de refroidissement (circulation d'eau, circuit refroidisseur à effet Peltier ...). Cependant, la chaleur restante induit des changements locaux d'indice dans le milieu laser provoquant des distorsions de phase sur les ondes se propageant dans le milieu.

Ces distorsions dues aux effets thermiques apparaissent pour des amplificateurs fonctionnant à des fréquences de répétition plus grandes que l'inverse du temps de relaxation thermique $1 / \tau_{t h}$ du milieu à gain. Pour un amplificateur du type $\mathrm{Nd}^{3+}$ :YAG on a $[2,5]$

$$
\tau_{\text {th }}=\frac{\mathcal{C} \rho}{4 K_{T}} r_{0}^{2}
$$

oì $\mathcal{C}$ est la chaleur spécifique, $\rho$ la densité et $K_{T}$ la conductivité thermique. $r_{0}$ est le 
rayon du barreau amplificateur. En prenant les valeurs suivantes pour un amplificateur Nd:YAG $[2,6]: \mathcal{C}=0,59 \mathrm{~W} \cdot \mathrm{g}^{-1} \cdot \mathrm{K}^{-1}, \rho=4,56 \mathrm{~g} \cdot \mathrm{cm}^{-3}, K_{T}=0,12 \mathrm{~W} \cdot \mathrm{cm}^{-1} \cdot \mathrm{K}^{-1}$ et un rayon typique de $r_{0}=3,5 \mathrm{~mm}$, on trouve un temps de relaxation thermique de 0,7 secondes. Ainsi les distorsions thermiques atteignent un état stationnaire à des taux de répétitions supérieures à quelques Hertz.

Nous considérons un barreau amplificateur de longueur $L$. Nous supposons que le milieu s'échauffe uniformément sous l'action de lampes flash et que $L>r_{0}$ de telle sorte que la répartition de la température est uniquement radiale. Le système de refroidissement périphérique du barreau maintient une température constante $T\left(r_{0}\right)$ à la surface dı barreau. L'équation à une dimension de conduction de la chaleur se met alors sous la forme [2]

$$
\begin{array}{r}
\frac{\mathrm{d}^{2} T}{\mathrm{~d} r^{2}}+\frac{1}{r} \frac{\mathrm{d} T}{\mathrm{~d} r}+\frac{4 \Delta T}{r_{0}^{2}}=0 \\
\Delta T=\frac{\eta_{T} P_{\text {pompe }}}{4 \pi L K_{T}}
\end{array}
$$

où $P_{\text {pompe }}$ est la puissance électrique moyenne des lampes flash et $\Delta T$ est la différence de température maximale entre le centre et la surface du barreau. $\eta_{T}$ est la fraction de la pompe transformée en chaleur et est typiquement égale à $\sim 3-5 \%$. En régime stationnaire, la température atteint, en tout point du barreau, une valeur définie par

$$
T(r)=T\left(r_{0}\right)+\Delta T\left[1-\left(\frac{r}{r_{0}}\right)^{2}\right]
$$

Il s'établit donc un gradient de température dans la section transverse du barreau. Tout d'abord ce gradient donne lieu à une contrainte mécanique. En effet, la partie la plus chaude, au centre, voit son expansion limitée par la partie périphérique, plus froide. Cette contrainte va induire de la biréfringence par effet photo-élastique. Cet effet va provoquer la dépolarisation de l'onde amplifiée. Par exemple, une onde polarisée linéairement dans le plan vertical, voit environ $20 \%$ de son énergie se transférer sur la composante horizontale après un passage dans un amplificateur Nd:YAG de $11,5 \mathrm{~cm}$ de long et fonctionnant à un cadence de $30 \mathrm{~Hz}$.

Le gradient de température est également à l'origine de l'effet de lentille thermique. En effet, l'indice dans le barreau peut se mettre sous la forme

$$
n(r)=n_{0}+\Delta n_{T}(r)+\Delta n_{\epsilon}(r)
$$

où $\Delta n_{\epsilon}$ correspond aux variations d'indice engendrées par l'effet photo-élastique d'origine thermique. Sous l'effet de $\Delta n_{\epsilon}$, le barreau agit comme une lentille bifocale avec une focale différente pour les composantes de polarisations radiale et tangentielle. $\Delta n_{T}$ est directement relié au gradient cle température radial dans le barreau. Cet effet est le plus important puisqu'il intervient à $90 \%$ dans la variation d'indice. $\Delta n_{T}$ est donné par

$$
\Delta n_{T}(r)=[T(r)-T(0)] \frac{\partial n}{\partial T}=-\Delta T\left(\frac{r}{r_{0}}\right)^{2} \frac{\partial n}{\partial T}
$$

où $\partial n / \partial T \simeq 8 \times 10^{-6} \mathrm{~K}^{-1}$ pour Nd:YAG. Il est utile d'introduire un indice non-linéaire thermique $n_{2}$ tel que $\Delta n_{T}(r)=-1 / 2 n_{2} r^{2} . n_{2}$ peut être mis sous la forme [5]

$$
n_{2}=\eta_{T} P_{\text {pompe }} C_{0}
$$




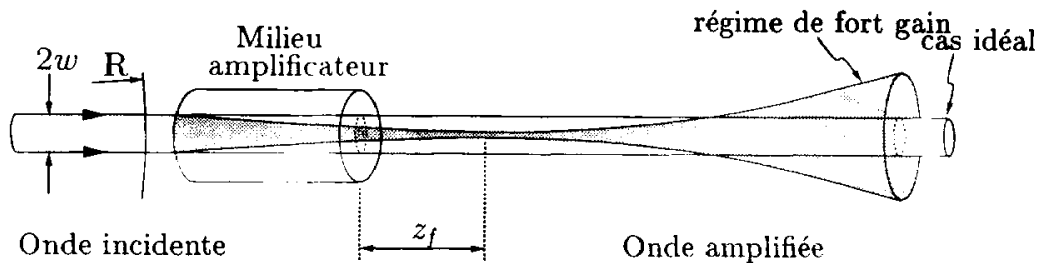

Figure 1: Représentation schématique de l'effet de lentille thermique dans un amplificateur laser solide.

$C_{0}$ étant une constante donnée par

$$
C_{0}=\frac{1}{2 \pi r_{0}^{2} L K_{T}} \frac{\partial n}{\partial T}
$$

Pour un barreau de Nd:YAG de longueur $L=11,5 \mathrm{~cm}, C_{0}=7,5 \times 10^{-3} \mathrm{~kW}^{-1} \mathrm{~cm}^{-2}$. Pour un faisceau incident de courbure $\mathcal{R}$ et de diamètre $2 w$ à l'entrée du barreau $(z=0)$, le changement de diamètre du faisceau le long de l'axe de propagation $z$ à l'intérieur du milieu, peut être calculé à partir de la matrice de transfert paraxiale suivante $[5,7]$

$$
\left(\begin{array}{cc}
A & B \\
C & D
\end{array}\right)=\left(\begin{array}{cc}
\cos \left(\sqrt{\frac{n_{2}}{n_{0}}} z\right) & \frac{1}{n_{0}} \sqrt{\frac{n_{0}}{n_{2}}} \sin \left(\sqrt{\frac{n_{2}}{n_{0}}} z\right) \\
-n_{0} \sqrt{\frac{n_{2}}{n_{0}}} \sin \left(\sqrt{\frac{n_{2}}{n_{0}}} z\right) & \cos \left(\sqrt{\frac{n_{2}}{n_{0}}} z\right)
\end{array}\right)
$$

puis, après le barreau, en considérant la matrice de transfert de propagation libre. Le faisceau subit un effet de focalisation, le milieu amplificateur se comportant alors comme une lentille convergente comme la montre la Figure 1.

La distance $z_{f}$ entre la sortie du barreau et le point de focalisation de l'onde est donnée par

$$
z_{f}=\frac{-w^{4}(B+A \mathcal{R})(D+C \mathcal{R})-\left(\lambda_{0} / \pi\right)^{2} D B \mathcal{R}^{2}}{w^{4}(D+C \mathcal{R})^{2}+\left(\lambda_{0} / \pi\right)^{2} D^{2} \mathcal{R}^{2}}
$$

On peut simplifier la matrice donnée par l'Eq. (8) en considérant que $n_{2}$ est petit par rapport à $n_{0}$. Ainsi la matrice de transfert de l'Eq. (8) se réduit à

$$
\left(\begin{array}{ll}
A & B \\
C & D
\end{array}\right)=\left(\begin{array}{cc}
1 & z / n_{0} \\
-n_{2} z & 1
\end{array}\right)
$$

Et dans l'approximation où la longueur d'onde $\lambda_{0}$ est suffisamment petite, $z_{f}$ peut se simplifier de la façon suivante

$$
z_{f} \simeq \frac{L+n_{0} \mathcal{R}}{n_{0}\left(L n_{2} \mathcal{R}-1\right)}
$$

Finalement dans l'approximation où $\mathcal{R}>>z_{f}$ et $\mathcal{R}>L / n_{0}$, le barreau laser peut être considéré comme une lentille de focale

$$
f \simeq z_{f} \simeq \frac{1}{L n_{2}}
$$

La Figure 2 montre l'effet de la lentille thermique en fonction de la puissance électrique $P_{\text {pompe }}$ des lampes flash. L'onde amplifiée se focalise d'autant plus près du barreau que 


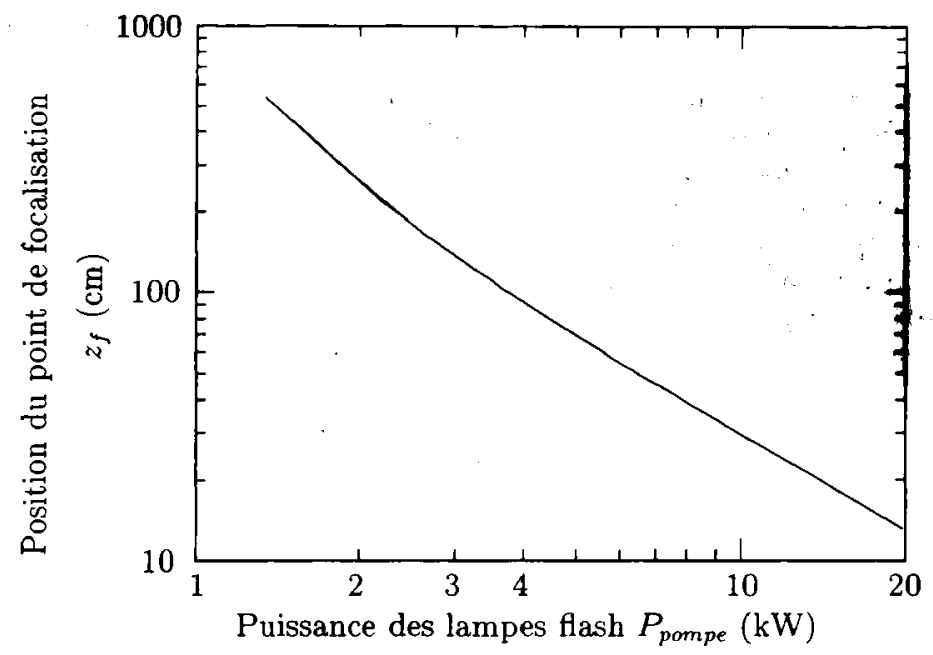

Figure 2: Mise en évidence de l'effet de lentille thermique: distance $z_{f}$ entre le point de focalisation de l'onde amplifiée et la sortie du barreau en fonction de la puissance électrique moyenne des lampes flash. La courbe a été tracée avec les paramètres suivants: $\eta_{T}=4 \%, L=11,5 \mathrm{~cm}, \mathcal{R}=3 \mathrm{~m}, w=2 \mathrm{~mm}$ et $\lambda_{0}=1,06 \mu \mathrm{m}$.

$P_{\text {pompe }}$ est grand. Il apparait clairement que les effets thermiques distordent fortement le barreau laser puisque ce dernier peut devenir équivalent à une lentille de focale $f \sim 10$ $\mathrm{cm}$. Pour ces calculs, nous avons considéré l'expression complète de $z_{f}$ donnée par l'Eq. (9).

\section{LA CONJUGAISON DE PHASE}

\subsection{Principe}

L'origine de la reconstruction de front d'onde optique remonte aux études de D. Gabor, en 1948 , qui aboutirent à l'invention de l'holographie $[8,9]$. Cette technique permet d'enregistrer sur un hologramme (du grec "holos", entier et "gram", message) une image qui peut ensuite être relue par un faisceau lumineux. Toutes les informations optiques de l'image sont alors transférées sur le front d'onde du faisceau. Cependant, l'holographie présente le défaut d'être un processus statique. L'inscription et la relecture d'un hologramme sont ell effet deux étapes bien distinctes et souvent longues à mettre en œuvre. Ainsi un hologramme donné ne peut pas corriger une onde se propageant dans des milieux (tels que l'atmosphère, les fibres optiques, les milieux amplificateurs ...) dont les distorsions peuvent varier dans le temps.

C'est en 1972 que B. Ya. Zel'dovich et ses collègues de l'Institut de Physique Lebedev, à Moscou, montrèrent expérimentalement qu'une onde "renversée temporellement" pouvait aussi être obtenue en réfléchissant un faisceau distordu sur un miroir Brillouin [10]. O. Y. Nosach et al. sont crédités de la première démonstration de correction de front d'onde utilisant la diffusion Brillouin stimulée [11]. Ils purent par cette technique diminuer la divergence d'un amplificateur laser à rubis. Ces auteurs introduisirent dans la littérature 
la notion d"inversion de front d'onde". Ce phénomène fut appelé "Conjugaison de Phase Optique". Cette expression a commencé à apparaitre dans la littérature vers $1977 / 78$, en connexion avec les études sur les processus non-linéaires tels que le mélange à deux ondes et le mélange à quatre ondes dégénéré [12-17]. L'analogie du mélange à quatre ondes avec l'holographie dynamique (ou en temps réel) fut alors rapidement établie. A cette époque, il fut également reconnu que le processus invoqué pour expliquer l' "holographie dynamique" était le même que l' "inversion de front d'onde" introduit par les Russes.

D'une façon générale, la conjugaison de phase optique permet de renverser la direction de propagation et la phase d'une onde optique [18-20]. Ce procédé peut être vu comme un miroir possédant des propriétés très particulières. Tout d'abord, un faisceau qui se réfléchit sur un miroir à conjugaison de phase, subit le même trajet que le trajet incident et ceci quel que soit l'angle du faisceau incident. La Figure 3(a) illustre cette propriété. Le miroir à conjugaison de phase produit l'inversion du vecteur $\vec{k}_{i}$, de telle sorte que le

(a)

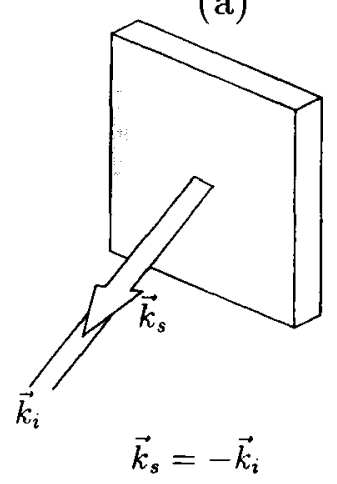

(b)

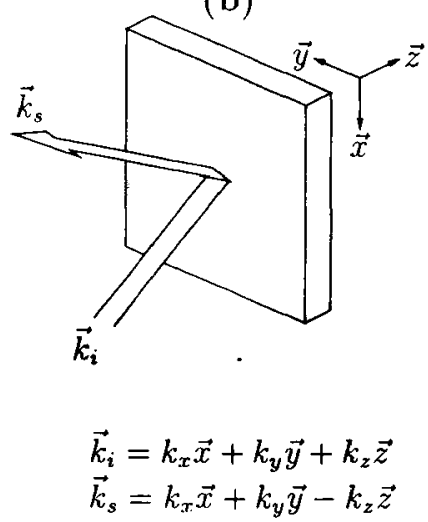

Figure 3: Comparaison de la reflexion d'une onde lumineuse sur (a) un miroir à conjugaison de phase et sur (b) un miroir classique.

faisceau réfléchi retourne sur le trajet du faisceau incident et ceci indépendamment de l'orientation du miroir à conjugaison de phase. En comparaison [Fig. 3(b)], un miroir plan classique change le signe de la composante du vecteur d'onde $\vec{k}_{i}$ normale au miroir alors que la composante tangentielle reste inchangée. Ainsi un faisceau incident peut être redirigé de façon aléatoire en tournant le miroir.

Outre le renversement du vecteur d'onde, le miroir à conjugaison de phase renverse également la phase de l'onde. Cette propriété s'avère être extrêmement utile pour corriger des fronts d'onde optique ayant subi des distorsions de phase. Comme le montre la Figure 4(a), l'onde plane incidente traverse un milieu aberrateur, une lentille divergente par exemple. Après réflexion sur un miroir à conjugaison de phase, le centre du faisceau voit

" transformer son avance de phase en retard (inversion de la phase). Ce retard va alors ètre annulé par le deuxième passage dans le milieu aberrateur. A la sortie de ce sytème l'onde retrouve sa structure d'onde plane et est ainsi complètement corrigée en phase. En comparaison, après réflexion sur un miroir classique [Fig. 4(b)], le centre du faisceau conserve son avance de phase. Un deuxième passage dans le milieu aberrateur va alors doubler les distorsions introduites lors du premier passage. De façon plus formelle, si l'on considère une onde optique se propageant dans la direction de l'axe $z$, son champ 


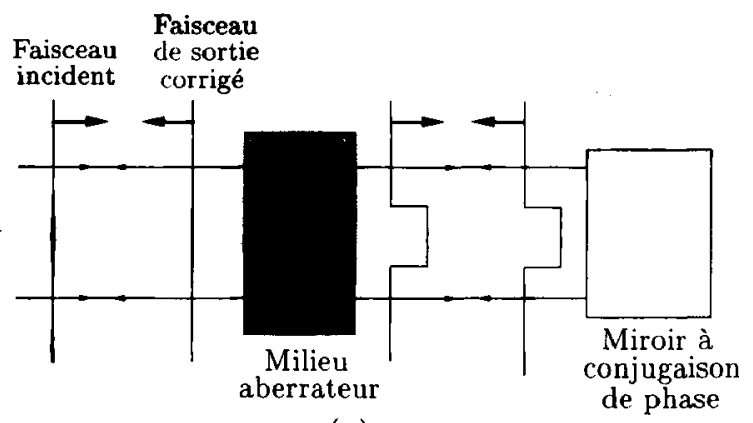

(a)

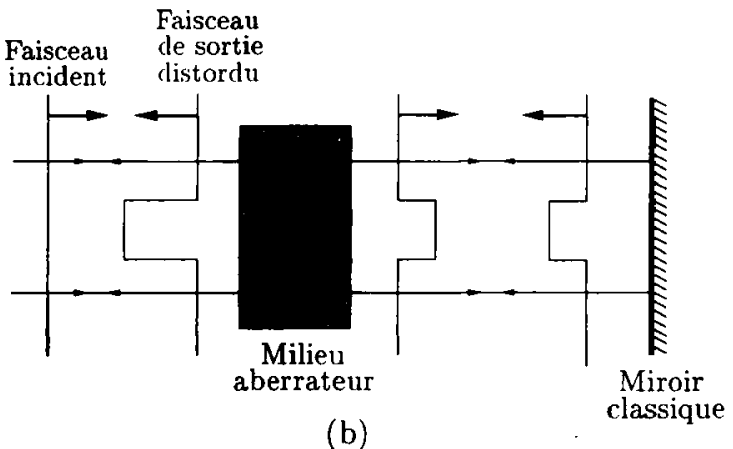

Figure 4: (a) Correction des distorsions de phase par un miroir à conjugaion de phase. (b) Doublement des distorsions de phase lorsqu'un miroir plan classique est utilisé.

électrique peut se mettre sous la forme

$$
\vec{E}=A \vec{e} \cos (\omega t-k z-\phi)
$$

oì $\omega$ est la pulsation de l'onde, $k$ l'amplitude du vecteur d'onde, $A$ l'amplitude du champ, $\vec{\rho}$ le vecteur unitaire définissant la polarisation de l'onde et $\phi$ sa phase. Nous avons vu qu1 un miroir à conjugaison de phase change le signe du vecteur $\vec{k}$ et celui de la phase $\phi$. Il en résulte que le champ de l'onde conjuguée est donnée par

$$
\vec{E}_{c} \propto A \vec{e}_{c} \cos (\omega t+k z+\phi)
$$

En fait l'expression de l'onde conjuguée peut directement s'obtenir à partir de l'Eq. (13) en changeant le signe de la variable de temps $t$. C'est pour cette raison que l'on parle souvent de renversement temporel lorsque l'on décrit le phénomène de conjugaison de phase.

Les applications qui découlent des propriétés des miroirs à conjugaison de phase sont nombreuses. Ainsi un faisceau de bonne qualité qui traverse une atmosphère turbulente peut décrire en sens inverse la même trajectoire s'il a été réfléchi par un de ces miroirs. Lorsque le faisceau revient à sa source, il n'est absolument pas déformé. De tels miroirs peuvent également être utilisés pour suivre la trace d'objets en mouvement, pour traiter 


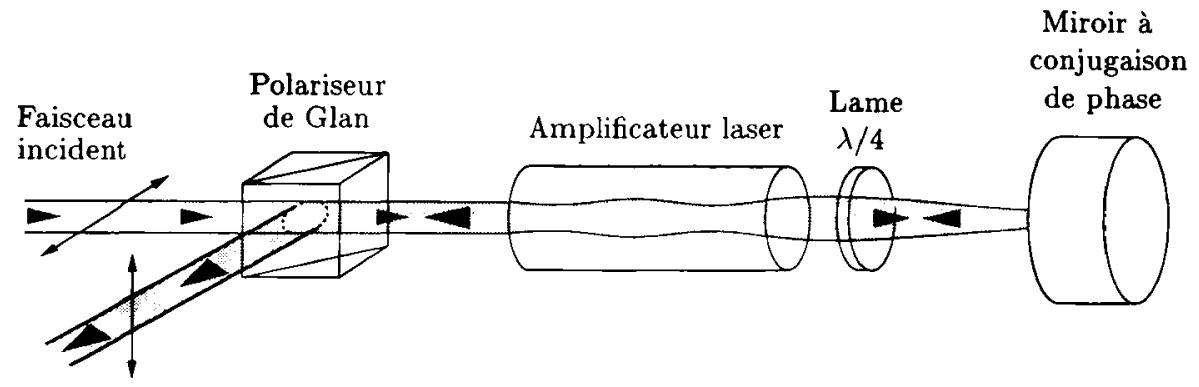

Faisceau amplifié

et corrigé en phase

Figure 5: Configuration MOPA ("Master Oscillator-Power Amplifier") en double passage avec un miroir à conjugation de phase.

des images, en interférométrie, dans les gyroscopes lasers, dans les systèmes de communication par fibres ou par satellites, et en lithographie [18-21].

Une des applications de la conjugaison de phase qui est certainement la plus étudiée et la plus prometteuse, est la correction d'une onde lumineuse se propageant dans un amplificateur laser. A cet effet, on utilise souvent le montage MOPA (de l'anglais "Master Oscillator-Power Amplifier") en double passage avec un miroir à conjugation de phase, comme le montre la Figure 5 [22,23]. Ce montage utilise un oscillateur maitre (non représenté sur la figure) délivrant un faisceau de faible énergie mais de très bonne qualité spatiale (typiquement limité par diffraction). Afin d'obtenir les fortes énergies lumineuses souhaitées, ce faisceau subit un passage dans un milieu laser à fort gain. Comme nous l'avons montré au Paragraphe précédent, la qualité du faisceau amplifié est dégradée par les distorsions de phase du milieu. Par réflexion sur le miroir à conjugaison de phase et un deuxième passage dans l'amplificateur, le faisceau finalement extrait est corrigé en phase et son front d'onde présente toites les caractéristiques de celui de l'onde incidente. Une lame quart d'onde $(\lambda / 4)$ est généralement utilisée pour tourner de $90^{\circ}$ la polarisation de l'onde amplifiée de telle sorte qu'elle puisse être extraite à l'aide d'un cube séparateur de polarisation (polariseur de Glan). De plus, pour corriger l'effet de dépolarisation induit thermiquement, ce montage peut être perfectionné en remplaçant la lame quart d'onde soit par deux lames demi onde montées en série avec un décalage de $45^{\circ}$ entre leurs axes neutres, soit par un rotateur de Faraday de $45^{\circ}[2,24]$.

\subsection{Les miroirs à conjugaison de phase}

Lo premier type de miroir à conjugaison de phase utilise le mélange à quatre ondes dégénéré [17]. Ce mécanisme non-linéaire peut se comprendre facilement par analogie avec l'holographie dont le principe est rappelé Figure 6. Dans un premier temps, un faisceau signal d'amplitude $S$ contenant des informations de phase interagit, au niveau d'une plaque photosensible, avec une onde plane de référence d'amplitude $A_{R}$. Les deux faisceaux étant cohérents, il va résulter de leur interférence, une répartition de la lumière qui dépend de la différence de leur phase relative en tout point de l'espace. Via un effet photochimique et après developpement de la plaque, cette distribution d'intensité va se trouver transposée sous forme de variation de l'indice de réfraction (créant ainsi un hologramme de phase) 


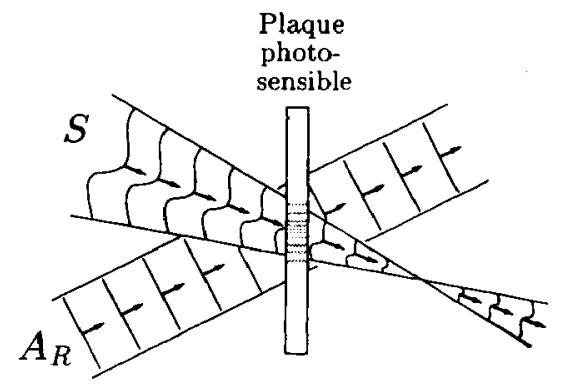

INSCRIPTION DE L'HOLOGRAMME

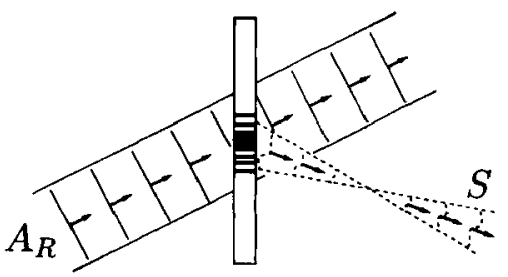

RELECTURE HOLOGRA PHIQUE

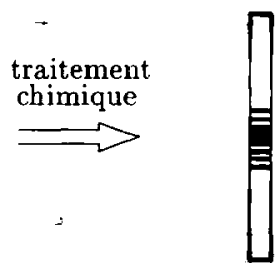

DEVELOPPEMENT DE L'HOLOGRAMME

$C \propto S^{*}$

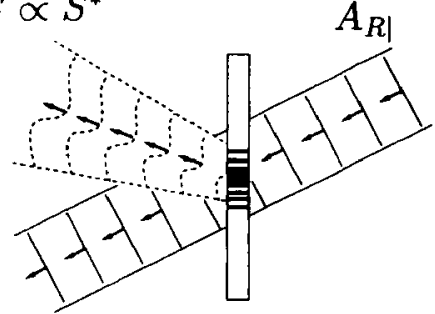

RELeCTURE DU CONJUGUÉ EN PHASE

Figure 6: Principe de l'holographie. L'enregistrement de l'hologramme se fait entre une onde plane de référence $A_{R}$ et un signal $S$. Après développement, l'hologramme peut être relu soit avec le faisceau de référence $A_{R}$ (relecture holographique), soit avec une seconde onde plane de référence d'amplitude $A_{R \mid}$ contra-propagative par rapport à $A_{R}$. Dans ce dernier cas, l'onde conjuguée en phase de $S$ est obtenue.

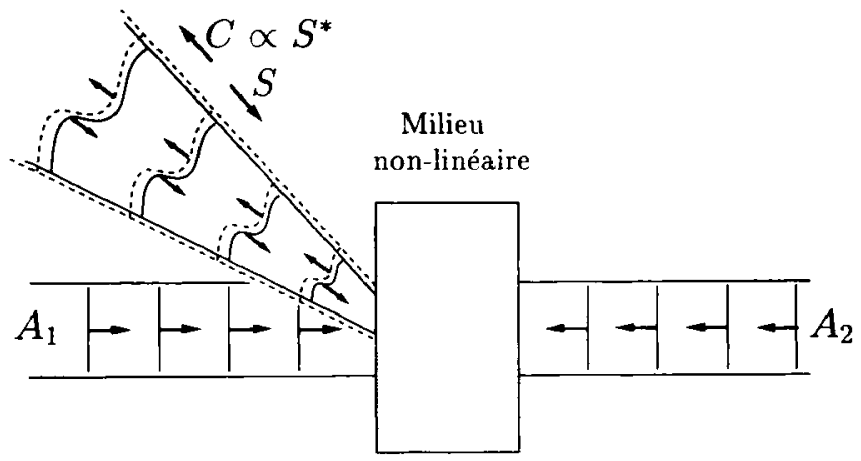

Figure 7: Miroir à conjugaison de phase utilisant le mélange à quatre ondes dégénéré dans un milieu non-linéaire dont l'indice complexe varie avec l'intensité. 


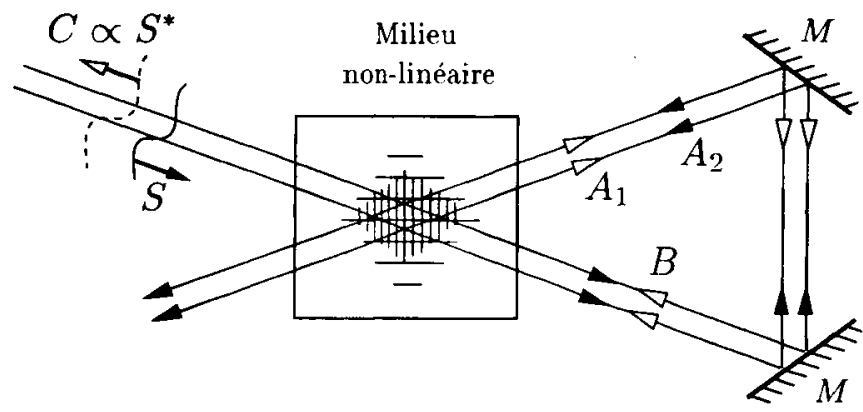

Figure 8: Miroir à conjugaison de phase auto-pompé fondé sur le principe du mélange à quatre ondes. La boucle de rétro-réaction est formée à l'aide de deux miroirs plans $(M)$.

on de variation de l'absorption (hologramme d'amplitude). C'est le processus d'écriture de l'hologramme. Dans un deuxième temps, l'hologramme peut être relu soit avec le faisceau de référence $A_{R}$ (relecture holographique), soit avec une seconde onde plane de référence d'amplitude $A_{R \mid}$ contra-propagative par rapport à $A_{R}$. Dans ce dernier cas, l'onde conjuguée en phase d'amplitude $C \propto S^{*}$ est obtenue. Dans le mélange à quatre ondes, toutes ces étapes se réalisent simultanément de façon dynamique et adaptative comme le montre la Figure 7. Le milieu non-linéaire dont l'indice varie avec l'intensité lumineuse tient lieu de support holographique. Les faisceaux pompes $A_{1}$ et $A_{2}$ du miroir à conjugaison de phase jouent le rôle des faisceaux de références $A_{R}$ et $A_{R \mid}$ de l'holographie classique. De plus, l'hologramme dynamique inscrit à un temps donné n'est pas irréversible. Il peut être effacé en éclairant uniformément le milieu non-linéaire ou en inscrivant un nouvel lologramme.

Le deuxième type de miroir à conjugaison de phase est également fondé sur le mélange à quatre ondes mais utilise la structure dite auto-pompée. Cette technique est schématisée sur la Figure 8 et présente l'avantage de ne pas utiliser de pompes extérieures comme dans le cas du mélange à quatre ondes classique. Le faisceau signal $S$ traverse une première fois le milieu non-linéaire, puis y est réinjecté à l'aide d'une boucle de rétro-réaction. Cette boucle peut être réalisée soit à l'extérieur du milieu par un jeu de miroirs diélectriques, soit à l'intérieur du matériau par réflexion sur ces faces. On peut alors distinguer deux types d'interaction.

Dans un premier cas, le faisceau signal $S$ interfère avec le faisceau $A_{2}$, créant un hologramme dynamique dans le milieu non-linéaire. Le faisceau $B$ issu initialement du bruit (diffusion du faisceau incident sur des impuretés, émission laser spontanée ...) en se diffractant sur l'hologramme donne le faisceau $A_{1}$. Après un aller dans la boucle, $A_{1}$ redonne $B$. Une oscillation constructive s'établit si la condition suivante est vérifiée

$$
\eta G p>1
$$

où $\eta$ est l'efficacité de diffraction de l'hologramme dynamique inscrit par $S$ et $A_{2}, T(<1)$ représente les pertes introduites dans la boucle. On voit qu'il est généralement nécessaire d'introduire un gain supplémentaire $G>1$ pour que la condition (15) soit satisfaite. Ce gain peut provenir d'un amplificateur laser introduit dans la boucle. Cette interaction est alors équivalente à un processus de mélange à quatre ondes où $A_{1}$ et $A_{2}$ représente les faisceaux pompes avant et arrière, $S$ le signal et $B$ le faisceau conjugué en phase au signal. 


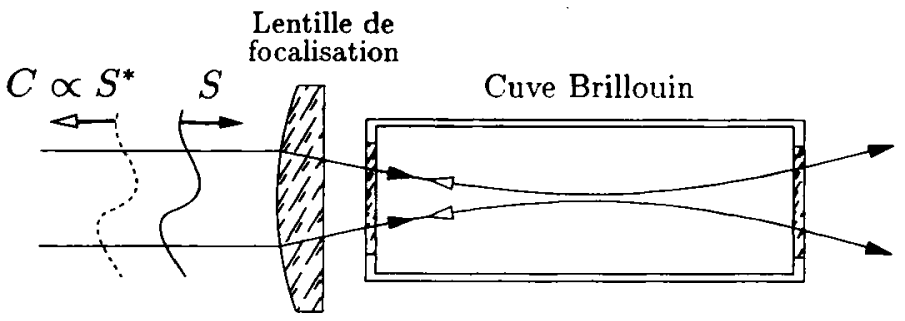

Figure 9: Miroir à conjugaison de phase auto-pompé utilisant la diffusion Brillouin stimulée dans un gaz à haute pression ou un liquide.

Dans le deuxième cas, le faisceau signal $S$ n'interfère pas avec le faisceau $A_{2}$. C'est le faisceau $A_{1}$ issu du bruit (diffusion du faisceau incident sur des impuretés, ...) qui en interférant avec $S$ crée un hologramme. Dans certaines conditions, le faisceau $A_{1}$ est amplifié par le signal $S$ via le processus de mélange à deux ondes. Après un aller dans la boucle, $A_{1}$ donne le faisceau $B$ qui va lui-même être amplifié par $A_{2}$. Ces amplifications successives aboutissent à la formation du faisceau conjugué en phase.

Finalement, le troisième type de miroir à conjugaison de phase utilise la diffusion Brillouin stimulée. Comme le mécanisme précédent, ce miroir est "auto-pompé" car le faisceau signal suffit seul à engendrer l'onde conjuguée. Ce processus non-linéaire étant efficace pour de fortes densités d'énergie, l'onde signal incidente doit être focalisée dans la cuve Brillouin contenant généralement un gaz à haute pression ou un liquide (voir la Figure 9). Ce phénomène débute par la présence dans le milieu d'ondes acoustiques spontanées résultant des fluctuations thermiques. Dans la région focale, l'onde incidente $S$ en interagissant avec ces ondes va diffuser dans toutes les directions. $S$ et ces ondelettes diffusées donnent naissance à des figures d'interférence. La force électrostrictive qui tend à concentrer les molécules dans les zones à grandes intensités va créer des réseaux d'indice dans le milieu. $S$ en diffractant sur ces réseaux, renforce les ondes diffusées. Comme l'onde conjuguée en phase présente le maximum de recouvrement spatiale avec $S$, c'est cette onde qui va s'établir préférentiellement au détriment de toutes les autres directions.

Les principaux matériaux non-linéaires utilisés comme miroirs à conjugaison de phase sont donnés dans le Tableau 1. Les mécanismes non-linéaires qu'ils emploient ainsi que leur domaine spectral et temporel d'utilisation sont également rappelés. Dans les paragraphes suivants, nous étudions plus précisément les mécanismes non-linéaires efficaces permettant d'obtenir une correction des aberrations des milieux lasers solides et plus particulièrement des lasers dopés au néodyme $\left(\mathrm{Nd}^{3+}\right)$ émettant dans le proche infrarouge.

\section{DIFFUSION BRILLOUIN STIMULEE}

Le premier mécanisme mis en jeu dans la diffusion Brillouin stimulée est l'interaction entre l'onde laser incidente et les ondes acoustiques spontanées présentes dans le milieu. Ces ondes acoustiques spontanées résultent des fluctuations thermiques existant dans un milieu à la température ambiante et ne dépendent évidemment pas de la présence de l'onde laser. Cette interaction spontanée va induire une diffusion aléatoire de l'onde incidente 


\begin{tabular}{|c|c|c|c|c|}
\hline $\begin{array}{l}\text { mécanisme } \\
\text { non-linéaire } \\
\text { mis en jeu }\end{array}$ & matérialıx & $\begin{array}{l}\text { type de miroir } \\
\text { à conjugaison } \\
\text { de phase }\end{array}$ & $\begin{array}{l}\text { longeurs d'onde } \\
\text { typiques } \\
\text { d'utilisation }\end{array}$ & $\begin{array}{c}\text { régime } \\
\text { d'utilisation }\end{array}$ \\
\hline \multirow{2}{*}{$\begin{array}{c}\text { effet } \\
\text { photoréfractif }\end{array}$} & $\begin{array}{c}\mathrm{BaTiO}_{3}, \mathrm{SBN}, \\
\mathrm{KNbO}_{3}, \mathrm{LiNbO}_{3}, \\
\mathrm{BSO}\end{array}$ & \multirow{2}{*}{$\begin{array}{l}\text { DFWM, } \\
\text { SPPC }\end{array}$} & $\begin{array}{c}\text { visible, } \\
\text { proche IR } \\
\text { (jusqu'à } \sim 1 \mu \mathrm{m} \text { ) }\end{array}$ & \multirow{2}{*}{$\begin{array}{c}\text { continu, } \\
\text { impulsionnel }\end{array}$} \\
\hline & $\begin{array}{c}\text { GaAs, } \\
\text { InP, CdTe:V }\end{array}$ & & $\begin{array}{c}\text { proche IR } \\
\text { (jusqu'à } \sim 1,5 \mu \mathrm{m} \text { ) }\end{array}$ & \\
\hline \multirow{2}{*}{$\begin{array}{l}\text { génération } \\
\text { de porteurs } \\
\text { libres }\end{array}$} & $\begin{array}{c}\overline{\mathrm{GaAs}}, \mathrm{Si} \\
\mathrm{CdTe}, \mathrm{CdSe}, \mathrm{CdS}\end{array}$ & \multirow[t]{2}{*}{ DFWM } & $\begin{array}{c}\text { visible, } \\
\text { proche IR }\end{array}$ & \multirow[t]{2}{*}{ impulsionnel } \\
\hline & $\mathrm{HgCdTe}, \mathrm{InSb}$ & & 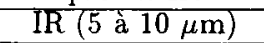 & \\
\hline $\begin{array}{c}\text { effet } \\
\text { thermique }\end{array}$ & $\begin{array}{l}\text { colorants, solvants, } \\
\text { cristaux } \\
\text { liquides }\end{array}$ & $\begin{array}{l}\text { DFWM, } \\
\text { SPPC }\end{array}$ & \multirow{3}{*}{$\begin{array}{l}\text { toutes } \\
\text { longueurs } \\
\text { d'onde }\end{array}$} & $\begin{array}{c}\text { continu, } \\
\text { impulsionnel } \\
\left(t_{p}>\mathrm{ns}\right)\end{array}$ \\
\hline \multirow{2}{*}{$\begin{array}{c}\text { effets de } \\
\text { réorientation } \\
\text { moléculaire }\end{array}$} & $\mathrm{CS}_{2}$ & DFWM & & $\begin{array}{l}\text { impulsionnel } \\
\left(t_{p} \sim \mathrm{ps}\right)\end{array}$ \\
\hline & $\begin{array}{l}\text { cristaux } \\
\text { liquides }\end{array}$ & $\begin{array}{l}\text { DFWM, } \\
\text { SPPC }\end{array}$ & & $\begin{array}{c}\text { continu, } \\
\text { impulsionnel }\end{array}$ \\
\hline \multirow{2}{*}{$\begin{array}{c}\text { saturation } \\
\text { de l'absorption } \\
\text { et } \\
\text { différence } \\
\text { de populations } \\
\text { atomiques } \\
\end{array}$} & $\begin{array}{l}\text { rubis, } \mathrm{Cr}: \mathrm{GdAlO}_{3}, \\
\text { verres dopés, } \\
\text { colorants, absorbants } \\
\text { saturables (BDN) }\end{array}$ & DFWM & \multirow{2}{*}{$\begin{array}{l}\text { dans la bande } \\
\text { d'absorption } \\
\text { du matériau } \\
(\mathrm{UV} \rightarrow \mathrm{IR} \text { ) }\end{array}$} & \multirow[t]{2}{*}{$\begin{array}{l}\text { continu, } \\
\text { impulsionnel }\end{array}$} \\
\hline & $\begin{array}{c}\text { gaz } \mathrm{SF}_{6} \text {, vapeurs } \\
\text { de } \mathrm{Na}, \mathrm{Rb}, \mathrm{Ba}\end{array}$ & $\begin{array}{l}\text { DFWM, } \\
\text { SPPC }\end{array}$ & & \\
\hline $\begin{array}{l}\text { saturation } \\
\text { du } \\
\text { gain }\end{array}$ & $\begin{array}{c}\text { lasers } \mathrm{Nd} \text { :YAG, } \\
\text { colorants }(\mathrm{Rl} 6 \mathrm{G}) \\
\text { vapeurs de cuivre, } \\
\text { gaz } \mathrm{CO}_{2}, \\
\text { excimère }(\mathrm{XeCl}, \mathrm{ArF})\end{array}$ & $\begin{array}{l}\text { DFWM, } \\
\text { SPPC }\end{array}$ & $\begin{array}{c}\text { dans la courbe } \\
\text { de gain du } \\
\text { matériau } \\
(\mathrm{UV} \rightarrow \mathrm{IR})\end{array}$ & impulsionnel \\
\hline $\begin{array}{l}\text { diffusion } \\
\text { Brillouin }\end{array}$ & $\begin{array}{c}\text { solides verre, quartz } \\
\text { fibres optiques } \\
\text { liquides } \mathrm{CS}_{2}, \mathrm{CCl}_{4} \text {, } \\
\mathrm{SnCl}_{4}, \mathrm{TiCl}_{4}, \mathrm{Fréon} \\
\text { gaz } \mathrm{N}_{2}, \mathrm{CH}_{4}, \mathrm{SF}_{6}\end{array}$ & SBS & $\begin{array}{c}\text { visible, } \\
\text { proche IR } \\
\text { (jusqu'à } \sim 2 \mu \mathrm{m} \text { ) }\end{array}$ & $\begin{array}{l}\text { impulsionnel } \\
\left(t_{p}<500 \mathrm{~ns}\right)\end{array}$ \\
\hline
\end{tabular}

Tableau 1: Principaux matériaux non-linéaires utilisés pour la conjugaison de phase optique. DFWM: mélange à quatre ondes dégénéré, SPPC: conjugaison cle phase auto-pompée fondée sur le mécanisme de mélange à cuuatre ondes, SBS: conjugaison de phase par diffusion Brillouin stimulée. $t_{p}$ : durée des impulsions; IR: infrarouge.

dans toutes les directions mais avec une efficacité extrêmement faible. Un calcul classique [19] montre que chacune de ces composantes de diffusion, parmi lesquelles se trouve l'onde conjuguée en phase, ne recueille que $e^{-30}$ fois la valeur de l'énergie incidente.

Le deuxième mécanisme, stimulé, va donc consister en une amplification sélective de la seule onde conjuguée en phase parmi toutes celles qui ont été diffusées par cette interaction spontanée. La diffusion stimulée s'opère elle-même en plusieurs étapes. Dans un premier temps, l'onde laser incidente et les ondes rétrodiffusées par l'interaction spontanée interfèrent, donnant naissance à des inhomogénéités de l'intensité. Si l'on prend l'exemple d'un gaz, la force électrostrictive tend à concentrer les molécules dans les zones à grandes intensités. Cette force transforme ainsi la figure d'interférence précédente en réseau de densité moléculaire, c'est à dire en une onde acoustique. Les ondes acoustiques ainsi créés 
s'atténuent en un temps $\tau_{B}$, caractéristique du temps de réponse de l'effet Brillouin. L'indice d'un gaz étant proportionnel à la densité moléculaire, un réseau d'indice reproduit donc la figure d'interférence à un décalage spatial près. La diffusion de l'onde incidente sur ces réseaux d'indice est alors un mécanisne stimulée de grande efficacité. Parmi toutes les ondes diffusées, seule l'onde conjuguée en phase cumule un gain important dans tout le volume d'interaction. Pour que ce mécanisme stimulé s'opère, l'onde incidente doit être suffisamment intense. La diffusion Brillouin stimulée est donc un phénomène à seuil.

\subsection{Détermination du seuil Brillouin}

Le modèle couramment utilisé pour décrire le processus de rétro-diffusion Brillouin stimulée permet d'aboutir à trois équations différentielles couplées donnant l'évolution du champ électrique $A_{L}$ du laser incident, du champ électrique $A_{C}$ de l'onde Stokes rétrodiffusée et de l'amplitude de l'onde sonore $Q$. Près du seuil, il est possible de considérer en bonne approximation que le champ incident $A_{L}$ n'est pas atténué par le processus de rétro-diffusion $\left(\left|A_{C}\right| \ll\left|A_{L}\right|\right)$. Dans ce cas, $A_{L}$ est constant et seulement deux équations couplées peuvent être conservées. Ces équations peuvent se mettrent sous la forme suivante [25]

$$
\begin{aligned}
& \frac{\partial A_{C}}{\partial z}-\frac{n}{c} \frac{\partial A_{C}}{\partial t}=g_{1} Q^{*} \\
& \frac{\partial Q}{\partial z}+\frac{Q}{\tau_{B}}=g_{2} A_{C}^{*}
\end{aligned}
$$

où $g_{1}$ et $g_{2}$ sont les coefficients de couplage, $n$ l'indice du mílieu, $c$ la vitesse de la lumière et $\tau_{B}$ est. la durée de vie du phonon.

Différents régimes temporels peuvent être identifiés en fonction de la durée de l'impulsion, $t_{p}$, par rapport à la durée de vie du phonon, $\tau_{B}[26]$.

\subsubsection{Régime stationnaire $\left(t_{p}>15 \tau_{B}\right)$}

Dans le régime stationnaire, les dérivées partielles par rapport au temps sont nulles et la résolution des équations (16) permettent de calculer la reflectivité $R$ du miroir non-linéaire

$$
R=r_{0} \exp \left(g I_{0} L\right)
$$

où $r_{0}$ est la reflectivité initiale créée par la diffusion spontanée, $g$ est le coefficient de gain Brillouin en régime stationnaire, $I_{0}$ est l'intensité du laser incident et $L$ est la longueur de l'interaction. Cette longueur est donnée par la longueur de Rayleigh, soit

$$
L=\frac{\pi w_{0}^{2}}{\lambda}
$$

où $w_{0}$ est le rayon du faisceau au point focal.

Le seuil Brillouin est franchi lorsque la diffusion stimulée devient plus importante (que le bruit de l'émission spontanée. Cette condition est. satisfaite lorsque le terme dans l'exponentielle de l'Eq. (17) vaut environ 30. Par conséquent l'intensité seuil, $I_{\text {seuil }}$ est donnée par

$$
I_{\text {seuil }}=\frac{30}{g L} \quad \text { (régime stationnaire) }
$$


Comme l'énergie seuil, $E_{\text {seuil }}$, peut s'exprimer en fonction de $I_{\text {seuil }}$ de la façon suivante

$$
E_{\text {seuil }}=t_{p} \pi w_{0}^{2} I_{\text {seuil }}
$$

où nous avons supposer une impulsion de forme temporelle rectangulaire. On trouve finalement

$$
E_{\text {seuil }}=t_{p} \lambda \overline{\frac{30}{g}} \quad \text { (régime stationnaire) }
$$

Cette dernière équation indique qu'en régime stationnaire le coefficient de gain Brillouin, $g$, doit être le plus grand possible pour optimiser le seuil.

\subsubsection{Régime transitoire $\left(t_{p}<15 \tau_{B}\right)$}

La résolution des équations couplées (16) en régime transitoire pour des impulsions de durée plus courte que la durée de vie du phonon permet de calculer la reflectivité $R$ du miroir non-linéaire. Ces calculs montrent que [19]

$$
R \propto r_{0} \exp \left(-2 \frac{t_{p}}{\tau_{B}}+2 \sqrt{2 g I_{0} L t_{p} / \tau_{B}}\right)
$$

Cette solution a été obtenue en faisant l'hypothèse que la reflectivité atteint son maximum au moins à la fin de l'impulsion et que l'impulsion de durée $t_{p}$ possède une forme temporelle rectangulaire. La différence essentielle entre l'Eq. (17) et l'Eq. (22) est que la reflectivité transitoire dépend du rapport $t_{p} / \tau_{B}$. Nous verrons que ceci a une influence très importante sur l'évolution du seuil Brillouin. Comme précédemment, le seuil est atteint lorsque le terme dans l'exponentielle de l'Eq. (22) vaut environ 30. Dans ce cas, l'intensité seuil, $I_{\text {seuil }}$ est donnée par

$$
I_{\text {seuil }}=\frac{\left(15+t_{p} / \tau_{B}\right)^{2}}{2 g L t_{p} / \tau_{B}} \quad \text { (régime transitoire) }
$$

En utilisant l'Eq. (20), on trouve finalement $[19,25]$

$$
E_{\text {seuil }}=\lambda \frac{15}{g}\left(7,5 \tau_{B}+t_{p}+\frac{t_{p}^{2}}{30 \tau_{B}}\right) \quad \text { (régime transitoire) }
$$

Il apparait que l'intensité seuil augmente très fortement en régime transitoire lorsque la durée de l'impulsion diminue. Ainsi

$$
\begin{aligned}
I_{\text {seuil }}\left(t_{p}=\tau_{B} / 10\right) & =30 \times I_{\text {seuil }} \text { (stationnaire) } \\
I_{\text {seuil }}\left(t_{p}=\tau_{B} / 100\right) & =400 \times I_{\text {seuil }} \text { (stationnaire) }
\end{aligned}
$$

En revanche, il faut approximativement toujours la même énergie pour franchir le seuil Brillouin en régime transitoire, et ceci quelle que soit la durée de l'impulsion incidente. En régime stationnaire, $E_{\text {seuil }}$ augmente linéairement avec la durée de l'impulsion [voir Eq. (21)]. 


\subsection{Paramètres importants}

Les équations (19), (21), (23) et (24) montrent que le gain Brillouin $g$ doit être le plus grand possible pour diminuer le seuil du mécanisme. le coefficient de gain Brillouin est donné par

$$
g=\frac{4 \pi^{2} \gamma_{e}}{c V_{S} \rho} \frac{\tau_{B}}{\lambda^{2}}
$$

Dans cette expression $\gamma_{e}$ est le coefficient electrostrictif, $\tau_{B}$ est la durée de vie du phonon, - la vitesse de la lumière et $\rho$ la masse volumique moyenne du milieu. Notons que $\gamma_{e}$ et $\tau_{B}$ sont proportionnels à $\rho$. Dans le cas d'un gaz, $\gamma_{e}$ et $\tau_{B}$ augmentent donc linéairemnent avec la pression. Le gain Brillotin augmente quadratiquement avec $\rho$ ou avec la pression.

La réflexion de l'onde incidente sur le réseau d'indice induit un décalage en fréquence $\Delta \nu_{\text {Brill }}$ entre faisceaux incident et réfléchi [19]

$$
\Delta \nu_{\text {Brill }}=2 \frac{V_{S}}{\lambda}
$$

où $V_{S}$ est la vitesse du son dans le milieu considéré et $\lambda$ la longueur d'onde du faisceau incident. Pour une onde excitatrice à $\lambda=1,06 \mu \mathrm{m}$, le décalage en fréquence Brillonin est cle 0,3 à $1 \mathrm{GHz}$ dans les gaz, de 2 à $3 \mathrm{GHz}$ dans les liquides et d'une dizaine de $\mathrm{GHz}$ dans les solides.

Parmi toutes les ondes diffusées par le processus spontané, celle conjuguée en phase est celle qui dispose du plus grand gain d'amplification cumulé sur tout le volume d'interaction. Cette amplification sélective explique pourquoi les miroirs Brillouin sont des miroirs à conjugaison de phase. En revanche, une intensité incidente trop importante, en réduisant le volume d'interaction réduit cette sélectivité et donc la qualité de la conjugaison de phase. L'intensité du faisceau incident doit donc être bien ajustée d'une part pour être au dessus du seuil et d'autre part pour permettre une bonne reconstruction du front d'onde conjugué en phase. En pratique, on choisit une intensité incidente entre 5 et 10 fois l'intensité seuil.

En résumé, les paramètres importants de cette interaction sont donc

- le gain Brillouin, $g$, (qui doit être le plus grand possible pour diminuer le seuil)

- la durée de vie des phonons acoustiques, $\tau_{B}$, qui quantifie le temps de réponse du miroir non-linéaire

- le décalage en fréquence Brillouin, $\Delta \nu_{\text {Brill }}$.

Le tableau 2 présente ces paramètres à la longueur d'oncle du laser Nd:YAG $(\lambda=1,06$ $\mu \mathrm{m})$ pour les milieux non-linéaires les plus connus.

La figure 10 montre une courbe expérimentale typique de réflectivité d'un miroir à conjugaison de phase Brillouin en fonction de l'énergie incidente. La reflectivité est ici définie comme le rapport énergétique entre le faisceau conjugué en phase et le faisceau incident. Le matériau utilisé est ici $\mathrm{du} \mathrm{SnCl}_{4}$ et le laser, un laser $\mathrm{Nd}$ :YAG déclenché fonctionnant à une longueur d'onde de 1,06 $\mu \mathrm{m}$. Dans ces conditions expérimentales, le seuil Brillouin est d'environ $2 \mathrm{~m} . J$. Pour des énergies supérieures, la réflectivité du miroir non-linéaire augmente rapidement puis atteint une valeur de saturation d'environ $60 \%$. Il 


\begin{tabular}{|c|c|c|c|c|}
\hline \multicolumn{2}{|r|}{ Matériaux } & $g(\mathrm{~cm} / \mathrm{GW})$ & $\Delta \nu_{\text {Brill }}(\mathrm{GHz})$ & $\tau_{B}(\mathrm{~ns})$ \\
\hline \multirow{4}{*}{ GAZ } & $\mathrm{CH}_{4}(120$ bars $)$ & 90 & 0,87 & 18,7 \\
\hline & Xe (40 bars) & 43 & 0,28 & 29,2 \\
\hline & $\mathrm{SF}_{6}$ (22 bars) & 25 & 0,25 & 17,1 \\
\hline & $\mathrm{N}_{2}$ (135 bars) & 30 & 0,7 & 22 \\
\hline \multirow{6}{*}{ LIQUIDES } & $\overline{\mathrm{CS}} \mathrm{S}_{2}$ & 130 & $\overline{3,3}$ & $\overline{5,2}$ \\
\hline & $\mathrm{CCl}_{4}$ & 4 & 2,8 & 0,6 \\
\hline & $\mathrm{C}_{2} \mathrm{Cl}_{3} \mathrm{~F}_{3}$ (Fréon 113) & 6 & 1,75 & 0,84 \\
\hline & $\mathrm{C}_{8} \mathrm{~F}_{18}$ & 6,5 & 1,4 & 1 \\
\hline & $\mathrm{SnCl}_{4}$ & 14 & 2,3 & 2 \\
\hline & $\mathrm{TiCl}_{4}$ & 20 & 3,3 & $1, \overline{7}$ \\
\hline
\end{tabular}

Tableau 2: Paramètres importants de l'effet Brillouin stimulé pour différents milieux non-linéaires à $\lambda=1,06 \mu \mathrm{m}$.

faut noter que les miroirs Brillouin utilisés dans des conditions optimales peuvent atteindre des réflectivités supérieures à $90 \%$.

\subsection{Effets non-linéaires parasites}

Le Tableau 2 montre que de nombreux gaz ou liquides possèdent les caractéristiques pour induire efficacement l'effet Brillouin stimulé. Pourtant, l'étude expérimentale de ces matériaux a permis de mettre en évidence des effets non-linéaires parasites qui peuvent très fortement diminuer, voir annuler, l'effet Brillouin recherché [27]. Les principaux mécanismes compétitifs à l'effet Brillouin peuvent être: l'ionisation du milieu, aussi appelée "claquage", la défocalisation thermique et l'effet Raman. Les milieux non-linéaires les mieux adaptés doivent donc être des matériaux qui possédent un coefficient de gain Brillouin élevé et dans lesquels les effets parasites limitatifs sont les plus faibles possibles.

Parmi les effets non-linéaires parasites, les effets thermiques sont certainement les plus contraignants. La première conséquence de l'effet thermique est la défocalisation de l'onde au voisinage du point focal qui a pour effet d'augmenter le seuil Brillouin. La défocalisation thermique d'une impulsion laser monomode est caractérisée par une énergie critique $E_{c r}$ pour laquelle la densité d'énergie au niveau du point focal est diminuée de moitié. Cette énergie critique est donnée par [28]

$$
E_{c r}=\frac{\rho C_{p} \lambda^{2}}{2 \pi(\partial n / \partial T)_{P} \alpha}
$$

où $n$ est l'indice du milieu, $C_{p}$ la chaleur spécifique, $T$ la température due à l'échauffement du milieu et $\alpha$ est le coefficient d'absorption. Cette formule permet de calculer l'absorption du milieu pour laquelle les effets thermiques apparaissent avant même le seuil Brillouin. Une application numérique dans le cas des liquides chlorés $\left(\mathrm{SnCl}_{4}, \mathrm{TiCl}_{4}\right.$ ou $\left.\mathrm{CCl}_{4}\right)$ donne $[28]$

$$
\alpha\left(\mathrm{cm}^{-1}\right) \leq \frac{10^{-9} \text { ou } 10^{-8}}{N t_{p}}
$$

où $N$ est la cadence de répétition et $t_{p}$ est la durée de l'impulsion. Pour une impulsion de durée $t_{p}=20$ ns et une cadence de répétition de $N=50 \mathrm{~Hz}$, le coefficient d'absorption 


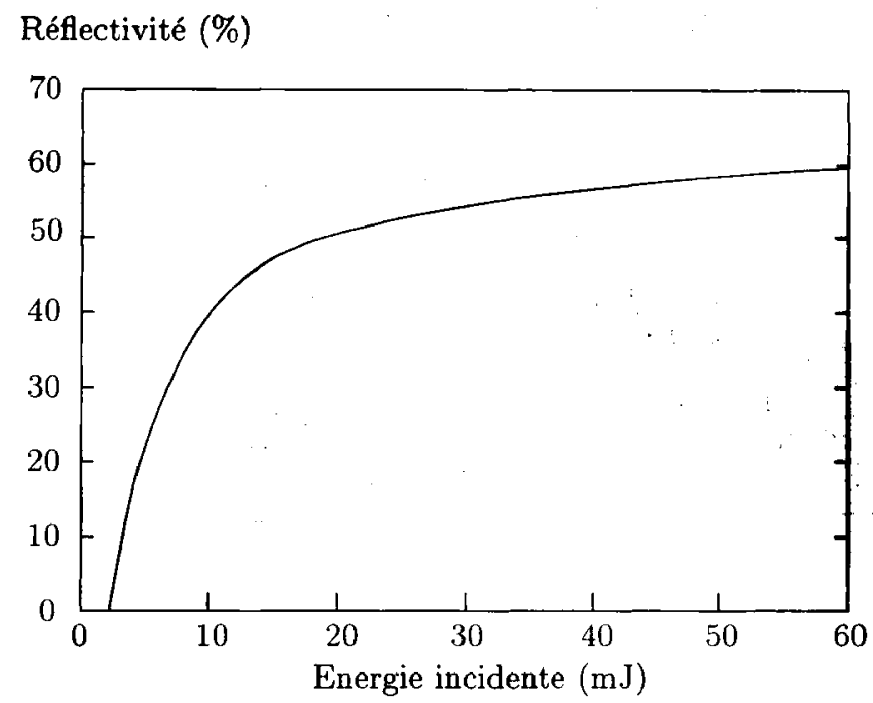

Figure 10: Réflectivité du miroir à conjugaison cle phase en fonction de l'énergie incidente par impulsion d'un laser Nd:YAG déclenché fonctionnant à 1,06 $\mu \mathrm{m}$. Le matériau Brillouin utilisé est du $\mathrm{SnCl}_{4}$.

du milieu doit être inférieur à environ $10^{-3} \mathrm{~cm}^{-1}$. Outre une absorption intrinsèque extrêmement faible, les milieux Brillouin doivent donc être parfaitement purifiés pour éviter l'absorption résiduelle par des impuretés.

\subsection{Influence des caractéristiques de la source sur le seuil Brillouin}

Pour un fonctionnement optimum du miroir Brillouin, le laser incident doit posséder une largeur de raie très étroite. Le seuil Brillouin le plus faible est donc obtenu lorsque le laser est monomode longitudinal. Lorsque le laser s'écarte de ce fonctionnement, le seuil Brillouin augmente. Il est à noter que dans ce cas, on peut également observer une baisse de la fidélité du miroir non-linéaire. Afin d'évaluer l'augmentation du seuil avec l'élargissement de la raie laser, nous avons utilisé la modélisation de la Ref. [29]. Le seuil Brillouin pour un laser possédant une raie de largeur $\Delta \omega$ est

$$
E_{\text {seuil }}(\Delta \omega)=E_{\text {seuil } 0}\left(1+\frac{4}{30} \frac{n \Delta \omega L}{c}\right)^{1 / 2}
$$

où $E_{\text {seuil } 0}$ est le seuil Brillouin pour un laser monochromatique $(\Delta \omega=0)$. Notons que cette expression a été calculée en considèrant le régime stationnaire. En bonne approximation, les augmentations du seuil en régime stationnaire et transitoire suivent une loi identique. On remarque que le seuil Brillouin augmente comme la racine carrée de la largeur de raie laser.

La qualité spatiale du faisceau incident peut également influencer très fortement le seuil Brillouin. Les conditions optimales de focalisation qui aboutissent au seuil Brillouin le plus faible sont en effet obtemues lorsque le faisceau est limité par diffraction (divergence 
$\left.\Theta_{0}\right)$. Pour un faisceau de divergence $\Theta$ qui n'est pas limité par diffraction $\left(\Theta>\Theta_{0}\right)$, le setuil Brillouin devient [28]:

$$
E_{\text {seuil }}(\Theta)=E_{\text {seu } l 0} \frac{\Theta}{\Theta_{0}}
$$

où $E_{\text {sezil } 0}$ est le seuil Brillouin pour un faisceau limité par diffraction. Cette formule montre que le seuil Brillouin augmente linéairement avec la divergence du faisceau incident.

\subsection{Exemple de réalisation}

Un laser du type oscillateur-amplificateur (MOPA) avec miroir à conjugaison de phase Brillouin est schématisé sur la Figure 11. Notons que plusieurs (en général deux) amplificateurs peuvent être utilisés et qu'un système de correction de la dépolarisation peut également être incorporé. Le miroir à conjugaison de phase et sa lentille de focalisation associée sont ici simplement placés après le premier passage du faisceau dans le (ou les) amplificateur(s). Comme nous l'avons évoqué dans les paragraphes précédents, le choix du milieu Brillouin est très important et doit tenir compte des caractérisatiques du faisceau incident sur la cellule (énergie par impulsion, cadence de répétition, distorsions, ...).

Quelques exemples de lasers à haute puissance et à haute qualité de faisceau:

- Laser Nd:YAG délivrant $1 \mathrm{~J}$ par impulsion à $100 \mathrm{~Hz}$ (100 W de puissance moyenne) entièrement pompé par diodes lasers [30]. Ce laser réalisé par TRW (USA) utilise un oscillateur Nd:YAG injecté pour un fonctionnement monomode longitudinal. L'amplificateur est un "slab" pompé par diodes lasers et la cellule Brillouin contient du Fréon 113. La qualité du faisceau en sortie du système est 1,1 fois la limite de diffraction.

- Laser Nd:YAP délivrant plus de $200 \mathrm{~W}$ de puissance moyenne avec une excellente qualité de faisceau réalisé à l'University de Berlin [31]. Le milieu Brillouin utilise du $\mathrm{CS}_{2}$.

Il est également possible de tirer profit du miroir à conjugaison de phase pour introduire dans le laser d'autres fonctions comme l'agilité angulaire du faisceau. Pour des applications industrielles comme le micro-usinage, il est nécessaire de dévier le faisceau rapidement avec une excellente précision de pointage. Pour réaliser cette fonction, on peut placer entre l'oscillateur et l'amplificateur un déflecteur acousto-optique. Le faisceau défléchi est ensuite amplifié une première fois puis rencontre le miroir à conjugaison de phase. Ce dernier renvoie le faisceau, quelle que soit sa direction, dans l'amplificateur. En sortie du système, on obtient un faisceau énergétique, défléchi et limité par diffraction [32].

On peut finalement mentionner qu'un laser à conjugaison de phase Nd:YAG est commercialisé par la société Coherent. Ce laser utilise un oscillateur en anneau monofréquence pompé par diodes lasers, deux barreaux amplificateurs pompés par lampes flash et une cellule Brillouin contenant du Fréon 113. Ce laser délivre des impulsions de $400 \mathrm{~mJ}$ à une cadence de $100 \mathrm{~Hz}$ et la qualité du faisceau est environ 1.5 fois la limite de diffraction.

Dans ces lasers, les matéridux non-linéaires utilisés sont soit des gaz maintenus à haute pression (100-200 bar), soit des liquides, bien souvent toxiques. D'un point de vue pratique, il est préférable d'utiliser des milieux à l'état solide qui présentent des avantages relatifs à leur compacité, facilité de manipulation et fiabilité. Leur utilisation est même indispensable pour certaines applications. 


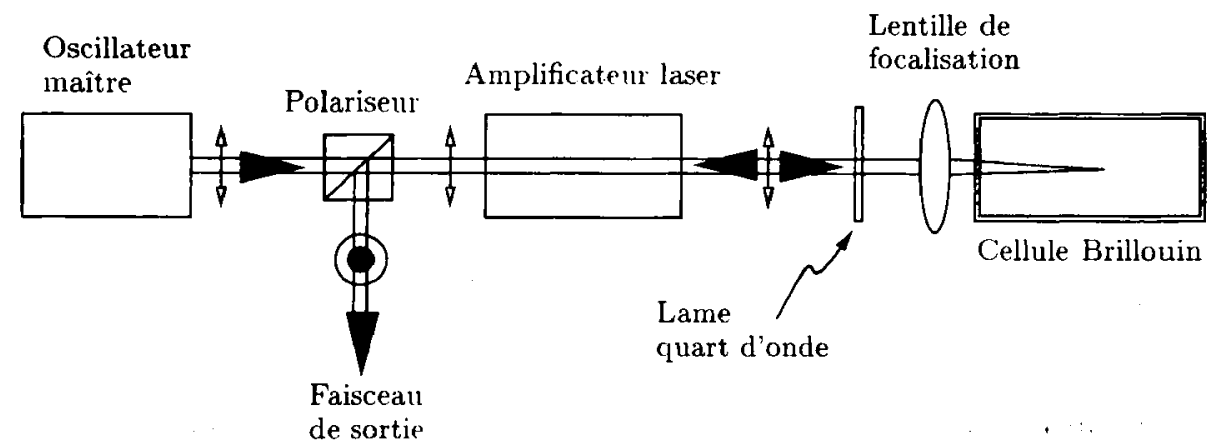

Figure 11: Réalisation d'un laser MOPA avec miroir à conjugaison de phase Brillouin.

Il peut être envisageable d'utiliser des matériaux solides pour réaliser des miroirs à conjugaison de phase Brillouin. Pour éviter tout phénomène non-linéaire parasite, ces matériaux doivent posséder un coefficient d'absorption très faible à la longueur d'onde d'utilisation. Dans le proche infrarouge, les verres optiques usuels (BK7) ou le quartz peuvent être employés. Cependant, leur coefficient de gain Brillouin est faible (environ $2-3 \mathrm{~cm} / \mathrm{GW}$ ) et leur seuil en énergie important (plusieurs dizaines de $\mathrm{mJ}$ ). Pour un fonctionnement correct, des impulsions d'énergie supérieure à $100 \mathrm{~mJ}$ doivent être focalisées dans le milieu, ce qui pose des problèmes de tenue an flux. Une solution peut consister d. utiliser des fibres optiques en verre ou en culartz très multimodes [31]. Comme le seuil Brillouin en intensité est inversement proportionnel au produit "coefficient de gain $\times$ longueur d'interaction", il est facile de pallier le faible coefficient de gain Brillouin en utilisant une fibre de plusieurs mètres. Des reflectivités de conjugaison de phase supérieures à 50 $\%$ ont ainsi été obtenues avec des impulsions de puissance crête de seulement quelques $\mathrm{kW}$ (dans un liquide, par exemple $\mathrm{SnCl}_{4}$, il faut plutôt des impulsions de puissance 1 MW). Des lasers MOPA avec une fibre à conjugaison de phase délivrant plus de $100 \mathrm{~W}$ de puissance moyenne ont ainsi pu être développés [33]. En revanche, la focalisation de l'onde incidente sur la face d'entrée de la fibre pose bien entendu des problèmes de positionnement du composant et de tenue au flux. Ce miroir à conjugaison de phase ne peut pas corriger des faisceaux trop distordus car toutes les fréquences spatiales ne rentrent pas dans la fibre.

\section{L'EFFET PHOTOREFRACTIF}

\subsection{Rappels de l'effet photoréfractif}

Parmi les matériaux solides envisageables, les cristaux photoréfractifs sont d'autres candidats intéressants. Un matériau photoréfractif associe deux propriétés : il est photoconducteur et il présente un effet électro-optique. La photoconduction, suite à une illumination 110 uniforme, permet l'établissement dans le matériau d'un champ électrique, dit champ rle charge d'espace, qui reproduit la figure en intensité mais qui est décalé d'une quantité $\phi$. Ce champ de charge d'espace est dû à la redistribution des charges photo-induites dans le matérian. Les porteurs de charges, par exemple des électrons, sont issus de centres donneurs ou "pièges photoréfractifs". Ces pièges sont dus à des défauts du cristal et leurs 


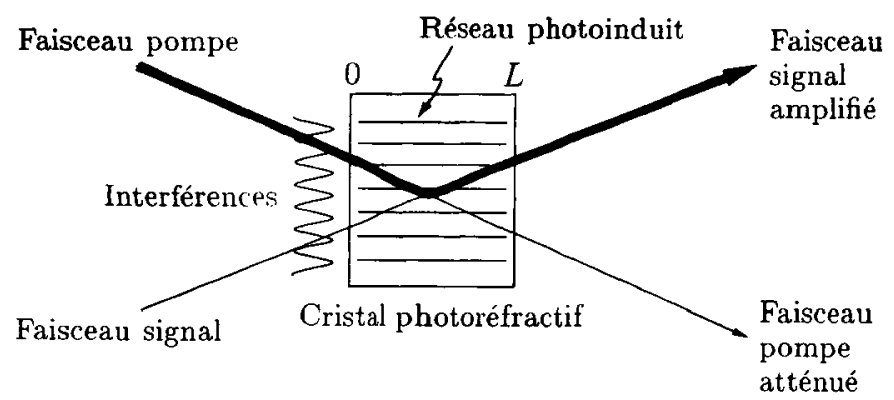

Figure 12: Principe du mélange à deux ondes dans un cristal photoréfractif.

niveaux d'énergie se trouvent dans la bande interdite. Ils sont excités dans les régions illuminées et passent dans la bande de conduction. Là, ils migrent par diffusion. Puis ils se combinent sur un centre accepteur, ou piège ionisé, dans les régions non illuminées. C'est donc cette séparation spatiale des charges qui induit le champ de charge d'espace. Ce dernier module par effet électro-optique la biréfringence du matériau. Ce changement local de l'indice du matériau reproduit donc la figure d'intensité à un décalage spatial $\phi$ près.

L'interaction de deux faisceaux cohérents dans un cristal photoréfractif permet un transfert d'énergie d'un faisceall vers l'autre. Ce phénomène appelé mélange à deux ondes est représenté sur la figure 12. Lorsque les deux ondes, d'amplitudes complexes $A_{p}$ et $A_{s}$, interfèrent dans le milieu non-linéaire, l'intensité totale peut s'écrire:

$$
I=\left|A_{p}\right|^{2}+\left|A_{s}\right|^{2}+\left[A_{p}^{*} A_{s} \exp (-i K r)+c . c .\right]
$$

où $K=4 \pi \sin \theta / \lambda$, où $\theta$ est le demi-angle entre les deux faisceaux à l'intérieur du cristal. La variation spatiale d'éclairement induit une variation de l'indice de réfraction qui s'écrit :

$$
n=n_{0}+\left(\frac{\Delta n}{2} \frac{A_{s}^{*} A_{p}}{\left|A_{p}\right|^{2}+\left|A_{s}\right|^{2}} \exp (-i K r) \exp (i \phi)+c . c .\right)
$$

ò̀ $\Delta n$ est le changement d'indice. En posant $A_{p}=\left(I_{p}\right)^{1 / 2} \exp \left(-i \psi_{p}\right)$ et $A_{s}=\left(I_{s}\right)^{1 / 2}$ $\exp \left(-i \psi_{s}\right)$, la résolution de l'équation de propagation permet d'aboutir aux systèmes d'équations couplées suivants:

$$
\begin{aligned}
\frac{\mathrm{d} I_{s}}{\mathrm{~d} z} & =\Gamma \sin \phi \frac{I_{s} I_{p}}{I_{s}+I_{p}}-\alpha I_{s} \\
\frac{\mathrm{d} I_{p}}{\mathrm{~d} z} & =-\Gamma \sin \phi \frac{I_{s} I_{p}}{I_{s}+I_{p}}-\alpha I_{p}
\end{aligned}
$$

et

$$
\begin{aligned}
\frac{\mathrm{d} \phi_{s}}{\mathrm{~d} z} & =\Gamma \cos \phi \frac{I_{p}}{I_{s}+I_{p}} \\
\frac{\mathrm{d} \phi_{p}}{\mathrm{~d} z} & =\Gamma \cos \phi \frac{I_{s}}{I_{s}+I_{p}}
\end{aligned}
$$




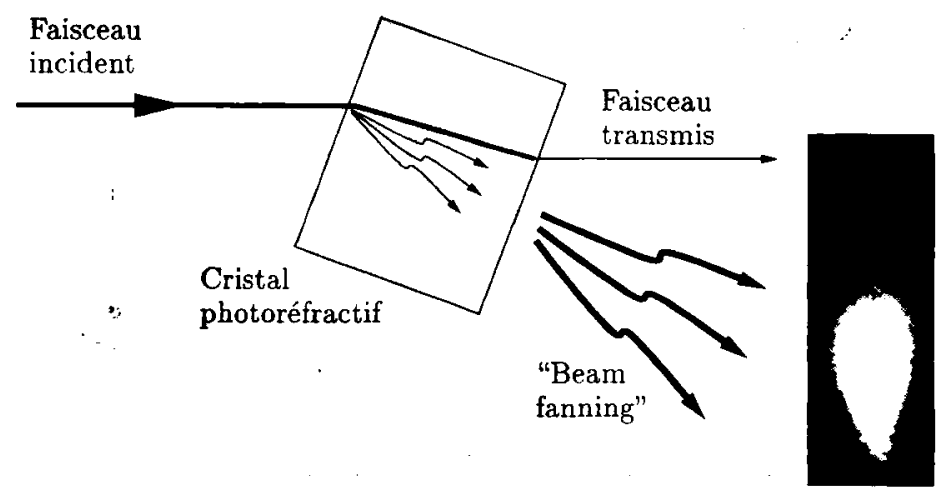

Figure 13: L'amplification du bruit dans un cristal photoréfractif ou "beam fanning".

où $\alpha$ est le coeficient d'absorption et $\Gamma$ le coefficient de gain photoréfractif donné par

$$
\Gamma=\frac{2 \pi \Delta n}{\lambda \cos \theta}
$$

Lorsque le matériau photoréfractif fonctionne en régime de diffusion (c'est à dire sans champ électrique appliqué), $\phi=\pi / 2$. Dans ce cas, on voit à travers les Eqs. (33) et (34) que le faisceau $A_{p}$ transfert son énergie au faisceau $A_{s}$ sans transfert de sa phase. Dans ces conditions, le gain deux ondes, $G=I_{s}(L) / I_{s}(0)$, peut se calculer d'après l'équation (33)

$$
G=\frac{1+\beta(0)}{\beta(0)+\exp (\Gamma L)} \exp (\Gamma L-\alpha L),
$$

où $\beta(0)=I_{p}(0) / I_{s}(0)$. Dans les matériaux photoréfractifs à fort gain tel que le Titanate de Barium $\left(\mathrm{BaTiO}_{3}\right)$, ce transfert énergétique peut être extrêmement efficace. En considérant un cristal de $0,5 \mathrm{~cm}$ d'épaisseur, un coefficient de gain $\Gamma=30 \mathrm{~cm}^{-1}$ et une absorption $a=1 \mathrm{~cm}^{-1}$, on obtient un gain deux ondes $G=2 \times 10^{6}$. Cette forte valeur de $G$ n'est généralement pas obtenue expérimentalement car l'interaction à deux ondes dans le $\mathrm{BaTiO}_{3}$ est perturbée par la présence d'un troisième faisceau appelé en anglais "beam fanning". Ce faisceau est issu de l'amplification de la diffusion de la pompe aux interfaces ou dans l'épaisseur du cristal via un couplage à deux ondes avec le faisceau pompe $\left(A_{p}\right)$ lui-même (voir figure 13). Cet effet indésirable dans une expérience de mélange à deux ondes, va jouer un rôle primordial dans la génération d'un faisceau conjugué en phase dans une géométrie auto-pompée.

\subsection{Conjugaison de phase auto-pompée dans un cristal photréfractif}

De la conjugaison de phase par mélange à quatre ondes peut être réalisée dans un cristal photoréfractif. Dans cette interaction un faisceau signal et deux faisceaux pompes contrapropagatifs interfèrent dans le cristal et engendrent un quatrième faisceau, le faisceau conjugué en phase. Pour l'application qui nous intéresse, cette interaction est difficilement utilisable. En revanche, les miroirs à conjugaison de phase auto-pompés présentent l'avantage de n'utiliser qu'un seul faisceau incident jouant le rôle de signal. Parmi les 


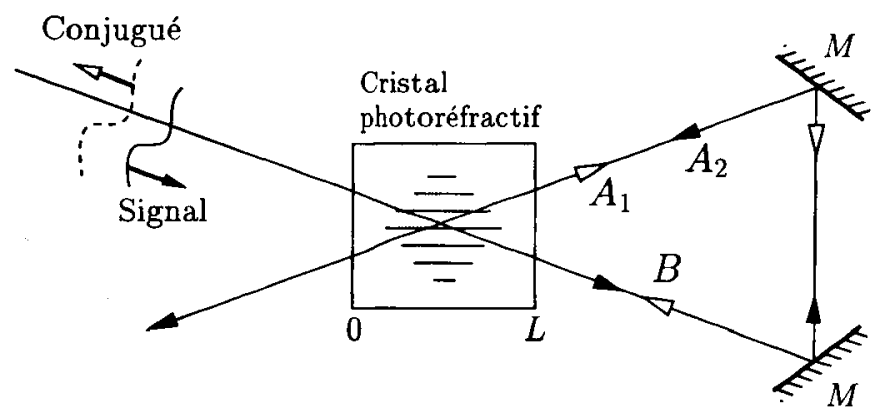

Figure 14: Conjugaison de phase auto-pompée en boucle externe dans un cristal photoréfractif. $\mathrm{M}$ : miroirs.

différentes géométries de conjugaison de phase auto-pompées, deux sont particulièrement intéressantes pour corriger les distorsions de phase d'un milieu laser.

La première géométrie est présentée sur la figure 14. Le signal traverse le cristal photoréfractif et y est renvoyé par l'intermédiaire d'une boucle optique réalisée au moyen de miroirs plans classiques. Le faisceau ainsi renvoyé est noté $A_{2}$. Un autre faisceau (noté $A_{1}$ ) est spontanément engendré dans le cristal grâce au phénomène de fanning. Après un aller dans la boucle, $A_{1}$ donne le faisceau $B$ qui va être à son tour amplifié par mélange à deux ondes entre $A_{2}$ et $B$. On se retrouve alors dans une configuration de mélange à quatre ondes où $A_{1}$ et $A_{2}$ jouent le rôle de faisceaux pompes avant et arrière, respectivement et où $B$ le conjugué en phase au faisceau incident. Le réseau photoréfractif utilisé pour la génération de la conjugaison de phase est le réseau en transmission inscrit simultanément par les couples de faisceaux (signal $\left.-A_{1}\right)$ et $\left(B-A_{2}\right)$. Le gain photoréfractif requis pour atteindre le seuil de fonctionnement du miroir à conjugaison de phase dépend de l'absorption du cristal et des pertes dues aux composants optiques de la boucle. Dans un modèle en ondes planes avec $\alpha=0$ et $\phi=\pi / 2$, le gain photoréfractif seuil est donné par [34]:

$$
\Gamma L=2 \frac{T+1}{T-1} \ln \left(\frac{T+1}{2 T}\right)
$$

où $T$ est la transmission de la boucle. $\Gamma L$ est égal à 2 pour une boucle sans pertes. Il faut noter que la réflectivité du miroir non-linéaire augmente rapidement avec le produit $\Gamma L$.

Lors de la réalisation de ce type de miroir à conjugaison de phase, deux problèmes peuvent se rencontrer : d'une part la stabilité de la réflectivité et d'autre part la fidélité de la conjugaison de phase. Nous présentons ici brièvement les techniques qui permettent d'obtenir un fonctionnement optimal du miroir non-linéaire. Pour assurer un fonctionnement stable de ce miroir à conjugaison de phase, on doit principalement éviter la formation des réseaux parasites en réflexion (réseaux formés entre les faisceaux $A_{2}$-signal, $B$ - $A_{1}$, $A_{2}-A_{1}, B$-signal) [35]. Si le laser utilisé possède une longueur de cohérence supérieure à la longueur de la boucle externe, il suffit de monter un des miroirs de la boucle sur une cale piezoélectrique pour rompre la cohérence du faisceau. Dans le plan de la boucle (plan de la figure 14), les conditions d'accord de phase imposent au faisceau conjugué en phase engendré à partir du bruit d'être une réplique fidèle du faisceau incident. En revanche, ces conditions d'accord de phase sont beaucoup moins strictes dans le plan vertical. Ceci a pour conséquence d'altérer la qualité du faisceau conjugué [36]. Pour pallier ce problème, une technique consiste à introduire dans la boucle un système qui réalise une rotation de 


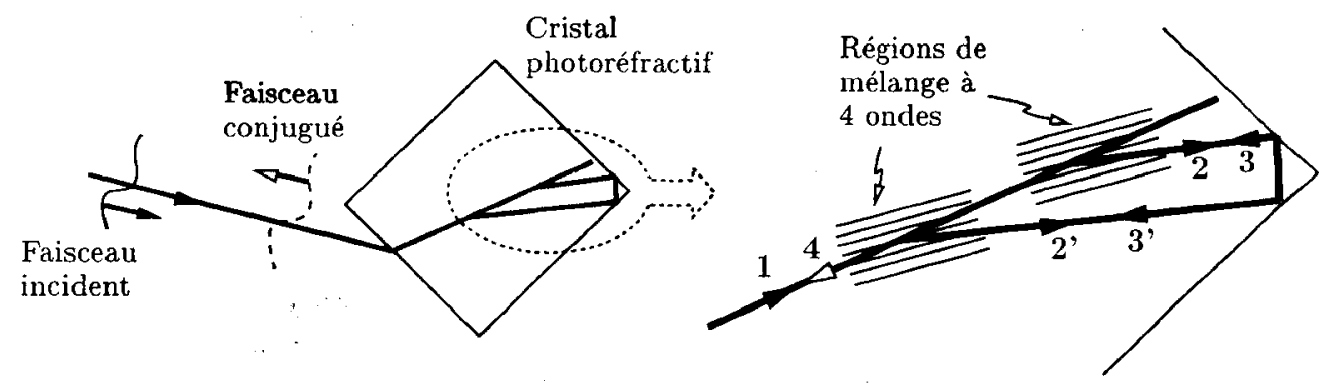

Figure 15: Conjugaison de phase auto-pompée par réflexion interne totale dans un cristal photoréfractif.

\begin{tabular}{c||c|c} 
& Boucle externe & Boucle interne \\
\hline Gain nécessaire & $\Gamma L>2$ & $\Gamma L>5$ \\
\hline Dimension du miroir & $\sim 10 \mathrm{~cm}$ & $<1 \mathrm{~cm}$ \\
\hline $\begin{array}{c}\text { Valeur de la } \\
\text { réflectivité }\end{array}$ & $\begin{array}{c}\text { ne dépend pas } \\
\text { du cristal }\end{array}$ & $\begin{array}{c}\text { peut dépendre de la } \\
\text { géométrie du cristal }\end{array}$ \\
\hline $\begin{array}{c}\text { Mise en place } \\
\text { du }\end{array}$ & $\begin{array}{c}\text { alignement précis des composants } \\
\text { optiques pour assurer un bon } \\
\text { miroir }\end{array}$ & $\begin{array}{c}\text { auto-alignement } \\
\text { du } \\
\text { fecouvrement entre les faisceaux }\end{array}$ \\
\hline $\begin{array}{c}\text { Caractéristiques } \\
\text { des distorsions }\end{array}$ & $\begin{array}{c}\text { ne corrige pas des aberrations } \\
\text { trop importantes }\end{array}$ & $\begin{array}{c}\text { peut corriger } \\
\text { de fortes aberrations }\end{array}$
\end{tabular}

Tableau 3: Comparaison des miroirs à conjugaison de phase auto-pompés photoréfractifs en boucles externe et interne.

la section des faisceaux de $90^{\circ}$ [37]. On peut également utiliser un télescope afocal dans la boucle.

La deuxième géométrie de conjugaison de phase auto-pompée utilise la formation d'une boucle à l'intérieur du cristal lui-même. Cette configuration ne nécessite aucun élément optique supplémentaire et présente donc l'avantage d'une grande compacité [38]. Comme dans le cas précédent, l'interaction démarre sur l'apparition du "beam fanning" dans le cristal. Pour certaines incidences du faisceau incident, la diffusion amplifiée se réfléchit sur les faces du cristal pour former une boucle interne au niveau d'un des coins du cristal comme le montre la figure 15. Le mécanisme de formation du faisceau conjugué en phase le plus couramment admis est celui des deux zones d'interaction [39]. Les faisceaux 2 et 2 ' engendrés à partir du beam fanning donnent respectivement les faisceaux 3 ' et 3 après réflexion sur les faces du cristal. Deux zones de mélange à quatre ondes apparaissent alors. Le signal (faisceau incident 1 ) et le faisceau conjugué en phase, 4 , sont communs à ces deux zones. Ce miroir non-linéaire nécessite une valeur de gain plus importante (environ $\Gamma L>5)$ que celle requise pour la configuration en boucle externe. Le tableau 3 compare les deux types de miroirs auto-pompés. 


\subsection{Lasers avec miroir à conjugaison de phase photoréfractif}

Pour réaliser une source laser à haute qualité de faisceau intégrant un miroir à conjugaison de phase photoréfractif, plusieurs paramètres sont à prendre en compte. Le choix du matériau photoréfractif est bien entendu crucial. Les impuretés ou le dopant éventuel du cristal photoréfractif doivent absorber à la longueur d'onde d'utilisation. En revanche, l'absorption ne doit pas être trop importante $\left(\alpha<1 \mathrm{~cm}^{-1}\right)$ pour éviter une limitation de la réflectivité ou tout échauffement du cristal. Les lasers en configuration MOPA fonctionnent par ailleurs en régime impulsionnel (impulsions de durée $\sim 10$ ns à une cadence de répétition $>10 \mathrm{~Hz}$ ). Les temps de réponse et la sensibilité des matériaux photoréfractifs ne permettant pas une réponse à une impulsion unique du miroir à conjugaison de phase, le faisceau conjugué s'établit après l'intégration de plusieurs impulsions. Les matériaux photoréfractifs fonctionnant en régime de diffusion (pour lesquels, $\phi=\pi / 2$ ) sont donc les seuls candidats possibles.

(a)

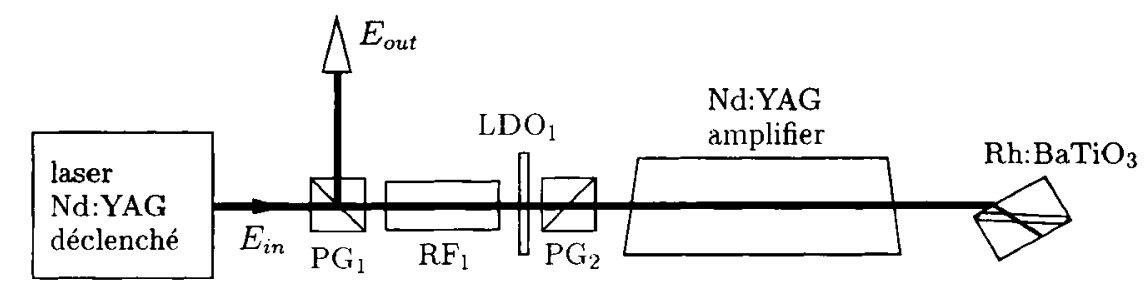

(b)

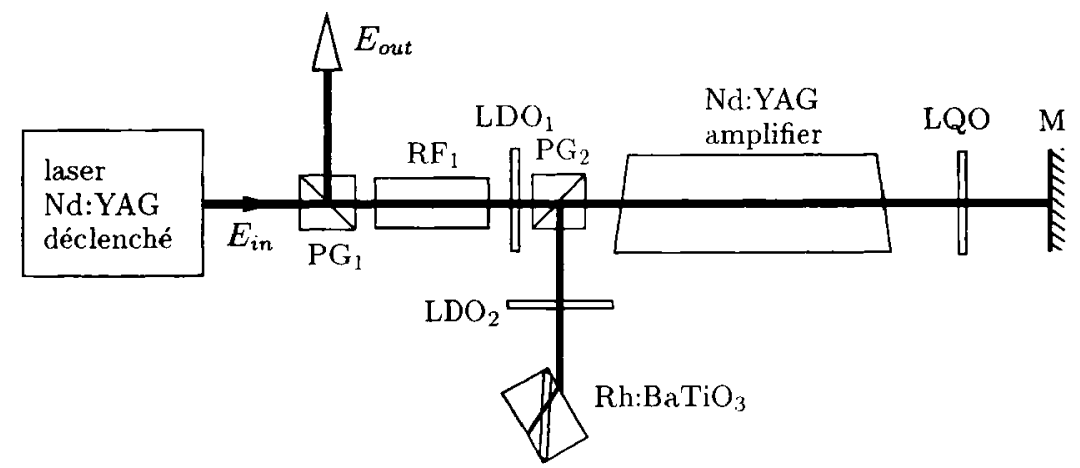

Figure 16: Architecture Oscillateur-Amplificateur (MOPA) avec miroir à conjugaison de phase photoréfractif. Géométries (a) 2 passages et (b) 4 passages. PG : polariseur de Glan; RF : rotateur de Faraday; LDO : lame demi-onde; LQO : lame quart d'onde; $\mathrm{M}$ : miroir plan.

Pour les lasers dopés au néodyme (Nd:YAG, par exemple) fonctionnant dans le proche infrarouge, le $\mathrm{BatiO}_{3}$ dopé au Rhodium $\left(\mathrm{Rh}: \mathrm{BatiO}_{3}\right)$ est un bon candidat [40]. Des réflectivités de conjugaison de phase de $55 \%$ en boucle interne et de $70 \%$ en boucle externe ont ainsi pu être démontrées avec des cristaux de $\mathrm{Rh}: \mathrm{BatiO}_{3}$ à la longueur d'onde des lasers Nd:YAG $(1,06 \mu \mathrm{m})$, ce qui est tout à fait suffisant pour envisager leur intégration dans un laser $[41,42]$. Le temps de réponse du cristal est de quelques minutes ce qui peut constituer une limitation lorsque les distorsions de phase du milieu laser évoluent rapi- 


\begin{tabular}{|c|c|c|}
\hline & Photoréfractif & Brillouin \\
\hline Milieu & + cristal & $\begin{array}{l}\text { - en général: liquide } \\
\text { ou gaz }\end{array}$ \\
\hline Dimension & $+<1 \mathrm{~cm}$ & $-20-50 \mathrm{~cm}$ \\
\hline $\begin{array}{c}\text { Caractéristiques } \\
\text { spectrales de la source }\end{array}$ & $\begin{array}{l}+ \text { pas de } \\
\text { contrainte }\end{array}$ & $\begin{array}{ll}- & \text { monomode } \\
& \text { longitudinal }\end{array}$ \\
\hline Temps de réponse & $-1-3 \mathrm{~min}$ & + qq. nanosecondes \\
\hline $\begin{array}{c}\text { Caractéristiques } \\
\text { spatiales } \\
\text { du } \\
\text { faisceau }\end{array}$ & 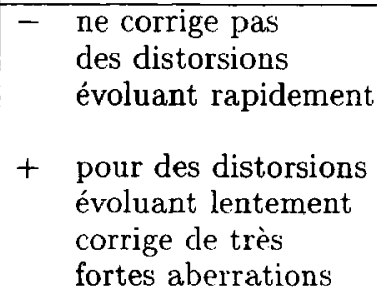 & $\begin{array}{l}+ \text { corrige des distorsions } \\
\text { pouvant changer d'une } \\
\text { impulsion à une autre } \\
-\quad \text { ne corrige pas } \\
\text { de fortes } \\
\text { aberrations }\end{array}$ \\
\hline
\end{tabular}

Tableau 4: Comparaison des miroirs à conjugaison photoréfractif et Brillouin. + : avantages; - : limitation.

dement. La tenue au flux du cristal $\left(\sim 15 \mathrm{MW} / \mathrm{cm}^{2}\right)$ est une autre contrainte à prendre en compte. En revanche, dans des régimes d'utilisation où le temps de réponse du cristal et la tenue au flux ne constituent pas un problème, l'utilisation d'un cristal photoréfractif comme miroir non-linéaire présente de nombreux avantages par rapport aux cellules Brillouin en terme de compacité, fidélité de conjugaison de phase, facilité d'utilisation et de manipulation.

Le schéma d'une source Nd:YAG avec miroir à conjugaison de phase photoréfractif est présenté sur la figure 16(a). Dans cette architecture, un oscillateur Nd:YAG déclenché délivre des impulsions de faible énergie, $E_{o s c}$, avec un profil spatial de bonne qualité. Un isolateur de Faraday, constitué de deux polariseurs de Glan $\left(\mathrm{GP}_{1}\right.$ et $\left.\mathrm{GP}_{2}\right)$, d'un rotateur de Faraday $\left(\mathrm{FR}_{1}\right)$ et d'une lame demi-onde $\left(\mathrm{WP}_{1}\right)$ permet de découpler l'oscillateur avec le faisceau de retour fortement amplifié. Le faisceau issu de l'oscillateur est donc amplifié une première fois dans l'amplificateur à fort gain. Le faisceau est alors réfléchi par le miroir non-linéaire pour subir un deuxième passage dans l'amplificateur. Le faisceau est ensuite extrait par réflexion sur le polariseur $\mathrm{GP}_{1}$. Ce faisceau amplifé possède toutes les qualités spatiales du faisceau issu de l'oscillateur. Comme on limite l'énergie incidente sur le cristal photoréfractif, toute l'énergie stockée dans l'amplificateur n'est pas extraite. Un moyen d'extraire une plus grande partie de cette énergie consiste à utiliser l'architecture de la figure 16(b) dans laquelle le faisceau subit 4 passages dans le milieu amplificateur [43]. Dans cette architecture, une lame quart d'onde (LQO) est utilisée en deux passages pour tourner de $90^{\circ}$ la polarisation de l'onde qui a subi une première traversée du milieu amplificateur. Aprés une deuxième amplification, l'onde est réfléchie par le polariseur $\mathrm{GP}_{2}$ et envoyé sur le miroir à conjugaison de phase. Une lame demi-onde $\left(\mathrm{WP}_{2}\right)$ renverse la polarisation pour optimiser l'interaction non-linéaire. L'onde conjuguée en phase ainsi renvoyée par le cristal photoréfractif subit deux passages supplémentaires dans l'amplificateur pour être finalement extraite du sytème au moyen du polariseur $\mathrm{GP}_{1}$. Une fois de plus, le faisceau de sortie est corrigé des distorsions de phase du milieu actif.

L'utilisation d'un cristal photoréfractif comme miroir non-linéaire permet donc la réalisation de sources lasers Nd:YAG à haute qualité de faisceau entièrement à l'état solide. C.e type d'architecture a récemment été validé avec un pompage par lampes flash basse 
cadence et par diodes laser de puissance haute cadence $[43,44]$. Dans ce dernier cas, des impulsions de $20 \mathrm{~mJ}$ à $200 \mathrm{~Hz}$ ont été obtenues (puissance moyenne $4 \mathrm{~W}$ ). Dans les mêmes conditions expérimentales, les performances de cette source en terme d'énergie extraite et de qualité de faisceau sont tout à fait comparables à celles obtenues avec un miroir à conjugaison de phase du type Brillouin. En revanche, pour atteindre des puissances plus élevées (10-200 W), les miroirs Brillouin restent encore les mieux adaptés. Une optimisation des matériaux photoréfractifs (dopage, taille des cristaux, temps de réponse) pourra certainement étendre leur domaine d'application. Le tableau 4 présente une comparaison des miroirs Brillouin et photoréfractif.

\subsection{Le "nettoyage de faisceau" par mélange à deux ondes}

Dans certains régimes temporels, il peut être difficile de réaliser des amplificateurs lasers possédant un gain suffisamment élevé pour être utilisable dans une architecture MOPA. C'est notamment le cas en régime continu ou en régime de très haute cadence ( $>10 \mathrm{kHz})$. La montée en puissance se fait alors dans l'oscillateur lui-même. La qualité spatiale du faisceau émis par de telles sources peut se trouver extrêmement dégradée par les distorsions thermiques qui interviennent dans le milieu actif intra-cavité. Une solution peut consister à remplacer un des miroirs de la cavité laser par un miroir à conjugaison de phase. En revanche, la réflectivité du miroir non-linéaire doit être proche de $100 \%$ pour diminuer les pertes de la cavité, ce qui constitue une limitation à l'emploi de cette technique. Une solution alternative consiste à utiliser le "nettoyage de faisceau" par mélange à deux ondes dans un cristal photoréfractif. Le principe du nettoyage de faisceau est représenté sur la figure 17. Comme on l'a déjà souligné au paragraphe 5.1, le mélange à deux ondes dans un cristal photoréfractif entre un faisceau intense, ou faisceau pompe, et un faisceau de faible intensité, ou faisceau signal, donne lieu à un transfert de l'énergie de la pompe sur le signal sans transfert de phase lorsque le décalage entre les réseaux d'interférence et d'indice vaut $\phi=\pi / 2$. En d'autres termes, si le signal est un faisceau d'excellente qualité spatiale mais peu intense et que le faisceau pompe intense est fortement distordu, cette interaction non-linéaire va permettre de transférer toute l'énergie du faisceau pompe sur le signal, ce dernier conservant toutes ses qualités spatiales [45].

L'architecture complète du laser incluant le module de nettoyage du faisceau est présentée sur la figure 18. L'oscillateur laser est schématisé par un milieu actif dans une cavité plan-convexe formée de deux miroirs. Le faisceau distordu, d'intensité $I_{0}$, issu du laser est

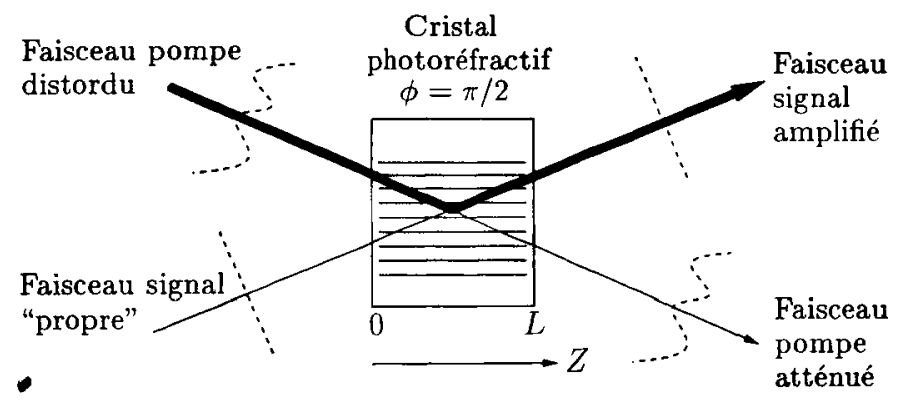

Figure 17: Principe du "nettoyage" de faisceau par mélange à deux ondes dans un cristal photoréfractif. 


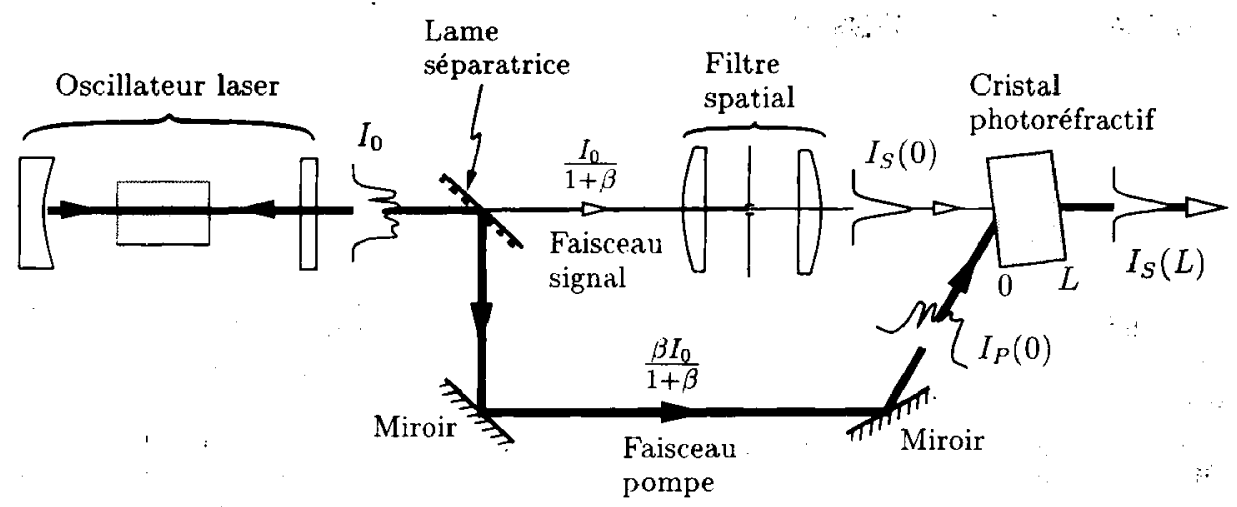

Figure 18: A mélioration de la luminance d'un oscillateur laser par mélange à deux ondes dans un cristal photoréfractif.

séparé en deux faisceaux au moyen d'une lame de verre séparatrice. Le premier faisceau, jouant le rôle de signal, est filtré au moyen d'un filtre spatial composé d'une plaque percée placée dans le plan focal de deux lentilles. Le diamètre du trou est choisi pour obtenir un faisceau limité par diffraction. Le signal, d'intensité $I_{S}(0)$ est alors mélangé dans le cristal photoréfractif avec le deuxième faisceau jouant le rôle de pompe, d'intensité $I_{P}(0)$. L'amplification du signal permet d'obtenir en sortie du cristal un faisceau limité par diffraction d'intensité $I_{S}(L)$. L'efficacité, $\mathcal{E}$, de cette technique peut être quantifiée par l'expression suivante:

$$
\mathcal{E}=\frac{I_{S}(L)}{I_{0}}
$$

En notant $\beta$ le rapport en intensité entre les faisceaux pompe et signal au niveau de la lame séparatrice, ce rapport juste avant le cristal devient

$$
\beta(0)=\frac{\beta}{T},
$$

où $T$ est la transmission du filtre spatial qui dépend directement de l'importance des distorsions du faisceau. En utilisant cétte valeur de $\beta(0)$ dans l'expression du gain deux ondes, $G$, donnée par l'équation (36), on peut calculer l'efficacité du système au moyen de la relation suivante:

$$
\mathcal{E}=\frac{T G}{1+\beta}
$$

Pour des valeurs de gain photoréfractif, $\Gamma L$, suffisamment importantes et une faible absorption, $\mathcal{E}$ peut tendre théoriquement vers $100 \%$, à condition d'optimiser la valeur de $\beta$. On peut noter que sans cette technique, un filtre spatial directement placé sur le faisceau issu de l'oscillateur limiterait la valeur de l'efficacité à $\mathcal{E}=T$. Cette technique a déjà été validée avec des lasers $\mathrm{Nd}$ :YAG continus et des cristaux photoréfractifs de $\mathrm{Rh}: \mathrm{BaTiO}_{3}$ sensible dans le proche infrarouge [46]. Notons que contrairement à la conjugaison de phase, l'efficacité de l'interaction à deux ondes est fortement tributaire de la stabilité et du contraste des franges d'interférences entre les faisceaux pompe et signal. Cette technique n'est donc utilisable que pour corriger les distorsions d'un laser possédant une cohérence spatiale suffisamment importante. 


\section{MELANGE D'ONDES DANS LE MILIEU LASER}

Une autre solution très attractive consiste à utiliser le milieu laser lui-même comme miroir non-linéaire. Il est en effet possible d'inscrire dans un amplificateur laser des réseaux ou des hologrammes dynamiques par saturation du gain. Cette technique proposée par R. L. Abrams et R. C. Lind en 1978 [47] et démontrée expérimentalement par R. A. Fisher et B. J. Feldman ainsi que A. Tomita en $1979[48,49]$ a fait l'objet récemment d'études plus approfondies $[50,51]$. Cette technique présente en effet de nombreux avantages. Le phénomène de saturation du gain laser est inhérent à tous les milieux lasers, par conséquent tous les matériaux lasers solides (Nd:YAG, Nd:YVO 4 , Ti:Saphir, ...) peuvent être utilisés. Le milieu laser étant lui-même le milieu non-linéaire, la longueur d'onde d'utilisation est automatiquement optimisée. Ce mécanisme ne possède aucun seuil en intensité et son temps de réponse, extrêmement court, est celui de l'émission stimulée. Enfin, comme toutes les ondes mises en jeu dans l'interaction non-linéaire sont amplifiées par le gain laser, on peut s'attendre à de très grandes réflectivités du miroir à conjugaison de phase ainsi formé.

Le principe de conjugaison de phase par mélange à quatre ondes dans un amplificateur laser est présenté schématiquement sur la Figure 19. Ce milieu laser doit être préalablement placé en inversion de population par pompage optique au moyen, par exemple, de lampes ou de diodes lasers de puissance. Au cours de cette interaction, deux faisceaux pompes contra-propagatifs et un faisceau signal se propagent dans le milieu non-linéaire. Ces trois faisceaux proviennent d'un même oscillateur laser et sont cohérents entre eux. Leur longueur d'onde est ajustée pour être à la résonance de la transition laser de l'amplificateur. Cette condition est automatiquement vérifiée lorsque le matériau laser de l'amplificateur est le même que celui utilisé pour l'oscillateur. La distribution non-uniforme de l'intensité lumineuse dans l'amplificateur, due aux interférences des trois ondes, inscrit un hologramme de gain dans le volume du matériau. En effet, dans les zones intenses, l'inversion de population décroît alors qu'elle reste élevée dans les zones sombres. La diffraction simultanée des faisceaux pompes sur cet hologramme de gain engendre une quatrième onde, conjuguée en phase du signal. Outre le gain de l'amplificateur, l'efficacité de cette interaction non-linéaire dépend aussi étroitement du taux de modulation de l'hologramme inscrit. Ce dernier peut être optimisé en ajustant correctement la densité d'énergie des ondes mises en jeu par rapport à la densité d'énergie de saturation $\left(U_{s a t} \simeq 500 \mathrm{~mJ} / \mathrm{cm}^{2}\right.$ pour le Nd:YAG) du milieu laser utilisé.

\subsection{Rappels sur les lasers}

Pour décrire et modéliser les interactions de mélanges d'ondes dans le milieu laser, nous considérons le modèle du laser à quatre niveaux de la figure 20 . Ce modèle s'applique aux lasers à néodyme fonctionnant vers $1,06 \mu \mathrm{m}$. Le pompage optique par lampes flash ou diodes lasers, permet d'exciter des atomes du niveau 0 fondamental vers le niveau 3 de pompage. Les atomes se relaxent ensuite de manière non-radiative vers le niveau haut de la transition laser (niveau 2). Le pompage permet de maintenir le niveau haut de la transition plus peuplé que le niveau bas créant ainsi une inversion de population. En traversant un tel milieu, une onde optique de fréquence $\nu$, d'amplitude initiale $A(0)$ subit une amplification laser de la forme:

$$
A(z)=A(0) \exp \left\{\frac{1}{2}[g(\nu)] z\right\} \times \exp \{-i[k+\phi(\nu)] z\}
$$

où $A(z)$ est l'amplitude de l'onde à la distance $z$ dans le milieu. $k=2 \pi / \lambda=2 \pi n / \lambda_{0}$ 


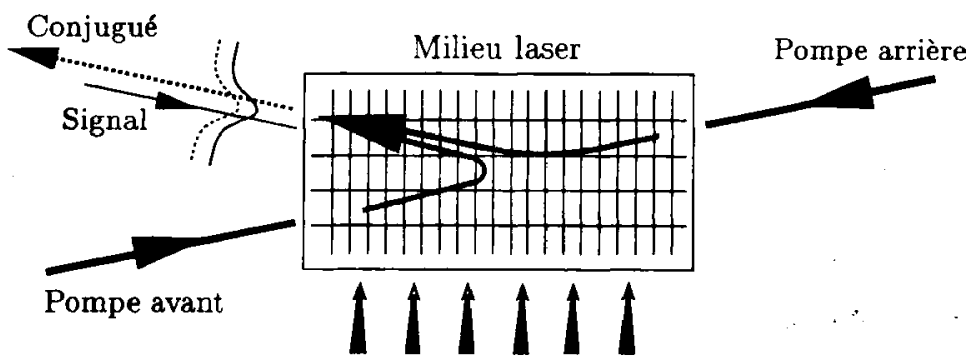

Pompage optique

(Flash -Diodes)

Niveau haut de la transition laser

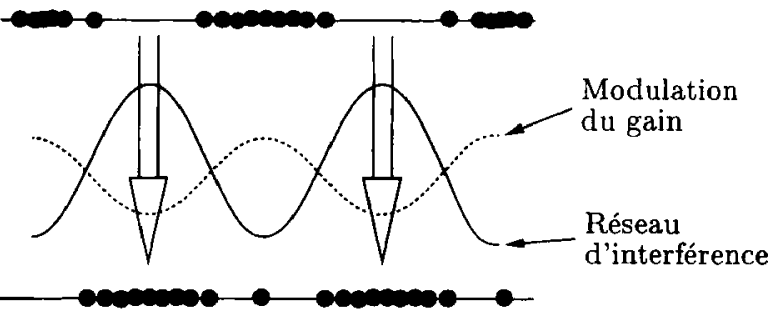

Niveau bas de la transition laser

Figure 19: Principe du mélange à quatre ondes par saturation de gain dans un milieu laser préalablement placé en inversion de population.

est, l'amplitude du vecteur d'onde dans le milieu, $n$ l'indice de réfraction. Le gain $g(\nu)$ et la phase $\phi(\nu)$ sont connectés à la susceptibilité atomique, $\chi$, des ions ou des molécules présents dans la matrice laser de la façon suivante [52]:

$$
g(\nu)=k \chi^{\prime \prime}(\nu) \text { et } \phi(\nu)=\frac{k}{2} \chi^{\prime}(\nu),
$$

où $\chi^{\prime \prime}$ et $\chi^{\prime}$ sont respectivement la partie imaginaire et la partie réelle de la susceptibilité atomique $\left(\chi=\chi^{\prime}+i \chi^{\prime \prime}\right)$. Pour des milieux qui présentent une raie laser à élargissement homogène, on $a$

$$
\begin{aligned}
\chi^{\prime \prime} & =\frac{g\left(\nu_{0}\right)}{k} \times \frac{1}{1+\delta^{2}}, \\
\chi^{\prime} & =\delta \chi^{\prime \prime},
\end{aligned}
$$

où $\nu_{0}$ est la fréquence de résonance du laser et

$$
\delta=\frac{2\left(\nu-\nu_{0}\right)}{\Delta \nu},
$$

avec $\Delta \nu$ la largeur totale à mi-hauteur de la raie laser. Dans l'équation (43), la valeur du coefficient de gain à la résonance, $g\left(\nu_{0}\right)$, est directement proportionnelle à l'inversion de population de la façon suivante

$$
g\left(\nu_{0}\right)=\sigma_{e}\left(\nu_{0}\right)\left(N_{2}-N_{1}\right),
$$



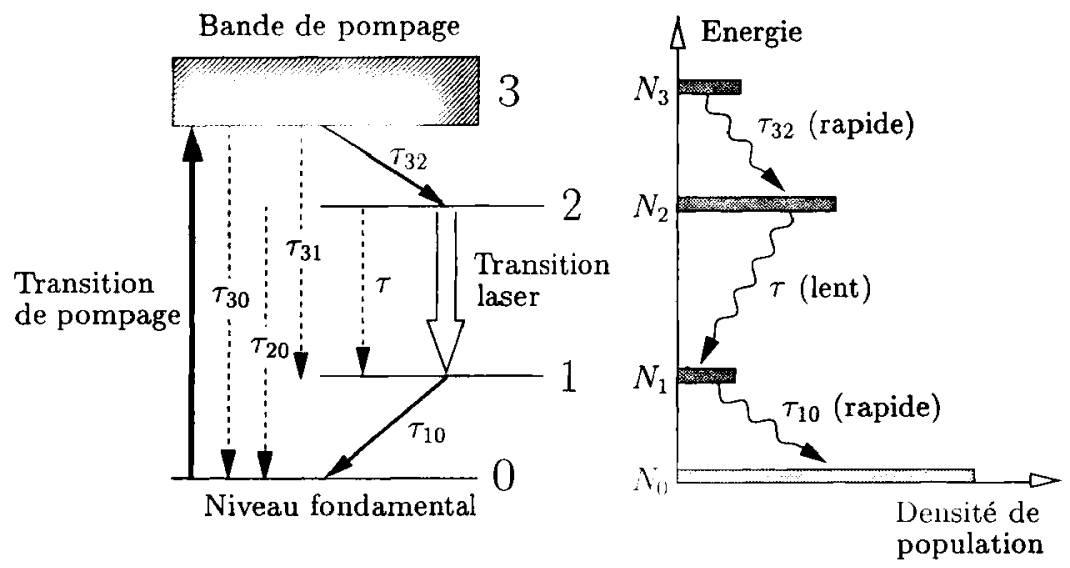

Figure 20: Représentation schématique du fonctionnement d'un laser à quatre niveaux.

où $\sigma_{e}$ est la section efficace d'émission et $N_{j}$ représente la densité de population du niveau $j$. Dans ce type de lasers, le niveau fondamental (niveau 0 de la Figure 20) est distinct du niveau bas de la transition laser (niveau 1). Ainsi, même avec un pompage modeste, l'inversion de population entre les niveaux haut et bas de la transition laser est facile à réaliser indépendamment du peuplement du niveau fondamental. Comme le niveau 3 de pompage se relaxe rapidement vers le niveau haut de la transition laser, on a $N_{3} \simeq 0$. Les équations de population des niveaux de la transition laser s'écrivent alors $[2,52]$

$$
\begin{aligned}
\frac{\partial N_{2}}{\partial t} & =-\frac{\sigma_{e}(\nu) I}{h \nu}\left(N_{2}-\frac{g_{2}}{g_{1}} N_{1}\right)-\frac{N_{2}}{\tau_{e f f}}-\frac{N_{2}}{\tau_{20}}+\eta_{P} W_{P} N_{0}, \\
\frac{\partial N_{1}}{\partial t} & =\frac{\sigma_{e}(\nu) I}{h \nu}\left(N_{2}-\frac{g_{2}}{g_{1}} N_{1}\right)-\frac{N_{1}}{\tau_{10}}+\frac{N_{2}}{\tau},
\end{aligned}
$$

où $I=|\vec{E}|^{2}$ est l'intensité de l'onde (ou des ondes) optique(s), à la fréquence $\nu$, se propageant dans le milieu laser et $\vec{E}$, le champ électrique total. $W_{P}$ est le taux de pompage. $\tau=\tau_{21}$ est le temps de relaxation de la transition laser où temps de fluorescence. $g_{j}$ indique le nombre d'états que peut prendre l'atome dans le niveau $j . g_{j}$ est appelé la dégénérescence du niveau $j$. Si les niveaux sont non-dégénérés, $g_{1}=g_{2}=1$. Le temps de relaxation spontanée $\tau_{\text {eff }}$ est donné par

$$
\tau_{e f f}=\frac{\tau}{1+\tau / \tau_{20}} .
$$

Bien souvent, la relaxation spontanée du niveau 2 vers le niveau 0 est négligeable. Dans ce cas, $\tau_{20} \sim \infty$ et $\tau_{\text {eff }} \sim \tau$.

D'après l'équation (46b), la population du niveau haut de la transition laser augmente grâce au pompage optique et diminue sous l'effet des émissions stimulée et spontanée. Quant à la population du niveau bas de la transition, elle augmente par émissions stimulée et spontanée et diminue par relaxation non-radiative vers le niveau fondamental. Cette lelaxation est caractérisée par la constante de temps $\tau_{10}$. Dans un laser à quatre niveaux idéal, cette relaxation se fait très rapidement de telle sorte que $\tau_{10} \simeq 0$. Ainsi, la densité 
de population $N_{1}$ est nulle. Dans ce cas, $N_{\text {tot }} \simeq N_{0}$ et l'inversion de population ( $\Delta N=$ $N_{2}-N_{1}$ ) devient $\Delta N \simeq N_{2}$. Avec ces approximations, on trouve finalement:

$$
\frac{\partial \Delta N}{\partial t} \simeq \frac{\partial N_{2}}{\partial t}=-\frac{\sigma_{e}(\nu) I}{h \nu} \Delta N-\frac{\Delta N}{\tau}+\eta_{P} W_{P} N_{t o t} .
$$

Dans la partie droite de l'équation (48), $-\sigma_{e} I \Delta N / h \nu$, décrit le phénomène d'émission stimulée, le deuxième terme, $-\Delta N / \tau$ est l'émission spontanée et le troisième terme, $R_{P} N_{\text {tot }}$, décrit le pompage optique, où $R_{P}=\eta_{P} W_{P}$ est le taux de pompage effectif.

Comme l'émission stimulée dépend de l'intensité $I$ de l'onde incidente et qu'il n'y a qu'un nombre fini d'atomes dans l'état excité, le gain du milieu diminue avec $I$. Ce phénomène est appelé la saturation du gain. On peut distinguer différents régimes de saturation suivant le comportement temporel de l'onde incidente. Ainsi en régime stationnaire, l'onde incidente est continue. On parlera également de régime stationnaire lorsque l'onde est une impulsion de durée $t_{p}$ très grande par rapport à la durée de vie de l'état excité $\tau(\simeq 230 \mu \mathrm{S}$ dans le cas du Nd:YAG). On parlera de régime impulsionnel ou régime transitoire, pour des impulsions de durée $t_{p}$ très petites par rapport à $\tau$. Dans la suite, on insistera plus particulièrement sur ce régime car dans la plupart des applications, des lasers Nd:YAG déclenchés délivrant des impulsions de queiques nanosecondes sont utilisés. Dans ce régime, comme $t_{p} \ll \tau$, on peut en bonne approximation, négliger l'émission spontanée et tout repompage optique éventuel. Dans ces conditions, l'équation (48) peut se réécrire de la manière suivante

$$
\frac{\partial \Delta N}{\partial t} \simeq \frac{\partial N_{2}}{\partial t}=-\frac{\sigma_{e}(\nu) I}{h \nu} \Delta N
$$

Pour une impulsion incidente dans le milieu à l'instant $t=0$, l'équation (49) aboutit à

$$
\Delta N(t)=\Delta N_{\text {init }} \exp \left[-\frac{\int_{0}^{t} I\left(t^{\prime}\right) d t^{\prime}}{U_{\text {sat }}}\right] .
$$

$\Delta N_{\text {init }}$ correspond à l'inversion de population initiale, juste avant que l'impulsion n'arrive. Dans l'équation (50), nous avons également introduit la densité d'énergie de saturation ou fluence de saturation définit par

$$
U_{s a t}(\nu)=\frac{h \nu}{\sigma_{e}(\nu)}
$$

L'expression de la susceptibilité atomique en régime transitoire à la résonance $\left(\nu=\nu_{0}\right.$, $\delta=0$ ) est finalement donné par [d'après les équations (43), (45) et (50)]:

$$
\chi=\frac{i \alpha_{0}}{k} \exp \left[-\frac{\int_{0}^{t} I\left(t^{\prime}\right) d t^{\prime}}{U_{s a t}}\right] .
$$

Dans cette équation, $\alpha_{0}(\nu)=\sigma_{e}(\nu) \Delta N_{\text {init }}$ est le coefficient de gain faible signal obtenu pour $U_{0} \ll U_{s a t}$, où $U_{0}=\int_{0}^{t_{p}} I d t$ est la densité d'énergie de l'impulsion incidente. 


\subsection{Mélange à quatre ondes dans le milieu laser}

Dans le cas du mélange à quatre ondes, le champ électrique total s'écrit:

$$
\vec{E}(z, t)=\sum_{j=\mathbf{1}}^{j=\mathbf{4}} A_{j}(z) \vec{e}_{j} \exp \left(-i \overrightarrow{k_{j}} \cdot \overrightarrow{r_{j}}+i \omega t\right),
$$

où $\overrightarrow{k_{j}}, \vec{e}_{j}$ et $\overrightarrow{r_{j}}$ sont respectivement le vecteur d'onde, le vecteur unitaire de polarisation et le vecteur direction unitaire de l'onde $A_{j}$. Comme le montre la figure $21, A_{1}$ et $A_{2}$ représentent les faisceaux pompes avant et arrière, $A_{3}$ le faisceau signal et $A_{4}$ le faisceau conjugué en phase. En injectant cette expression dans l'équation de propagation dérivée des équations de Maxwell:

$$
\Delta \vec{E}-\frac{n^{2}}{c^{2}} \frac{\partial^{2} \vec{E}}{\partial t^{2}}=\frac{1}{\epsilon_{0} c^{2}} \frac{\partial^{2} \vec{P}^{a t}}{\partial t^{2}}
$$

on aboutit à un système de quatre équations couplées dans lesquelles chaque onde est couplée avec les autres par l'intermédiaire de coefficients non-linéaires dépendant euxmêmes de l'intensité des ondes. Dans l'équation (54), $\vec{P}^{a t}=\epsilon_{0} n^{2} \chi \vec{E}$ est la polarisation atomique. Dans le cas où tous les faisceaux sont polarisés de la même manière (mélange à quatre ondes avec faisceaux copolarisés), l'expression des coefficients non-linéaires est complexe. Nous ne traiterons ici que le cas où les deux faisceaux pompes sont polarisés orthogonalement. Si le signal est polarisé comme le faisceau pompe avant, un réseau de gain en transmission est inscrit dans le milieu laser, le faisceau pompe arrière en se diffractant sur ce réseau crée le faisceau conjugué comme indiqué sur la figure 21 . Le faisceau conjugué est alors polarisé orthogonalement au signal et au faisceau pompe avant. Dans ce cas, l'équation (52) devient:

$$
\chi(\mathbf{r}, t)=\frac{i \alpha_{0}}{k} \times \exp \left[-U_{S}(z, t)-U_{M}(z, t) \cos (K y)\right]
$$

où $K=2 k \sin \theta$ est le module du vecteur réseau, $U_{S}$ et $U_{M}$ sont donnés par [51]:

$$
\begin{aligned}
U_{S}(z) & =\sum_{j=1}^{j=4} \int_{0}^{t} \frac{\left|A_{j}(z)\right|^{2}}{U_{\text {sat }}} d t \\
U_{M}(z) & =2 \int_{0}^{t} \frac{A_{1}(z) A_{3}(z)+A_{2}(z) A_{4}(z)}{U_{\text {sat }}} d t
\end{aligned}
$$

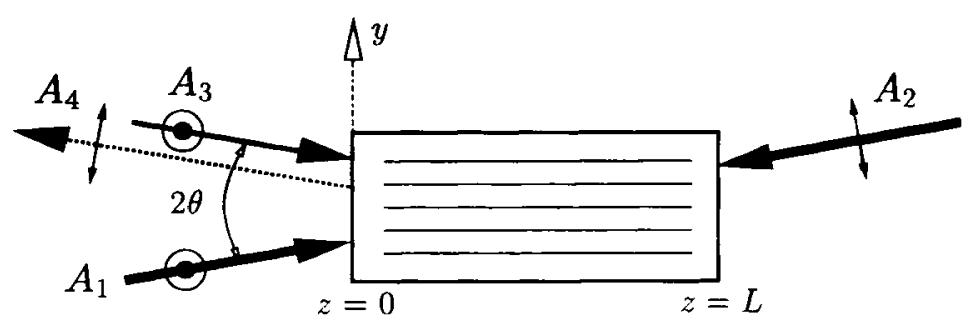

Figure 21: Mélange à quatre ondes dans un amplificateur laser avec faisceaux pompes polarisés orthogonalement. 


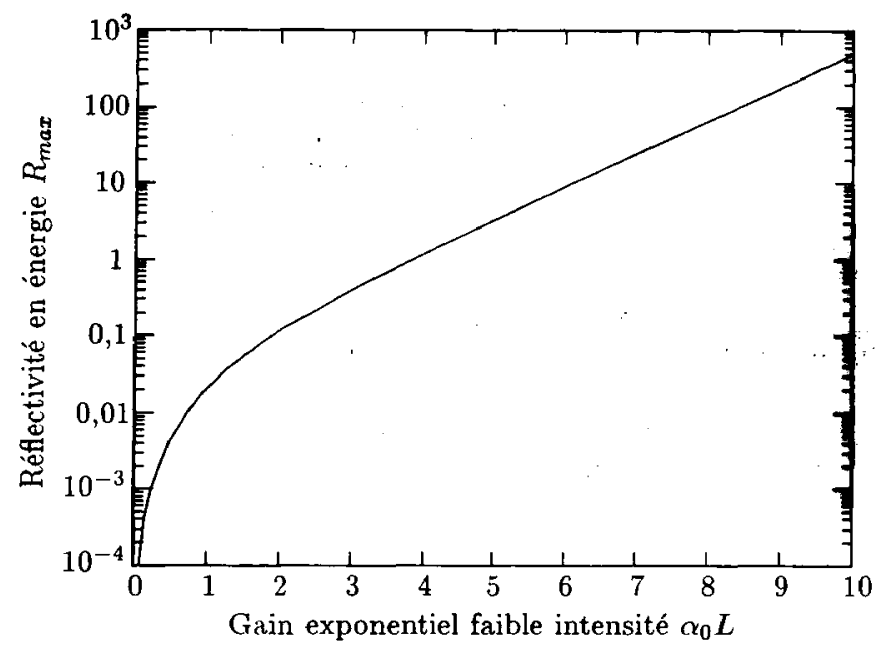

Figure 22: Réflectivité du miroir non-linéaire par mélange à quatre ondes dans un milieu laser en fonction du gain laser.

$\chi$ peut être développée en série de Fourier de la façon suivante:

$$
\chi=\sum_{n=-\infty}^{+\infty}(-1)^{|n|} \gamma_{|n|} \exp (-i n K \dot{y})
$$

où les coefficients non-linéaires $\gamma_{n}$ sont donnés par [51]

$$
\gamma_{n}(z, t)=\exp \left(-U_{S}\right) \mathcal{I}_{n}\left(U_{M}\right)
$$

$\mathcal{I}_{n}$ étant la fonction de Bessel hyperbolique à l'ordre $n$. En injectant la susceptibilité atomique dans l'équation de propagation (54), on obtient les équations différentielles couplées entre les ondes $A_{1}, A_{2}, A_{3}$ et $A_{4}$ dans l'hypothèse de l'enveloppe lentement variable:

$$
\begin{aligned}
\frac{d A_{1}(z)}{d z} & =\gamma_{0} A_{1}-\gamma_{1} A_{3}, \\
-\frac{d A_{2}(z)}{d z} & =\gamma_{0} A_{2}-\gamma_{1} A_{4}, \\
\frac{d A_{3}(z)}{d z} & =\gamma_{0} A_{3}-\gamma_{1} A_{1}, \\
-\frac{d A_{4}(z)}{d z} & =\gamma_{0} A_{4}-\gamma_{1} A_{2} .
\end{aligned}
$$

La résolution numérique de ces équations permet de calculer la forme temporelle de l'impulsion conjuguée, son énergie et la réflectivité du miroir à conjugaison de phase définie comme le rapport en énergie des faisceaux conjugué et signal en $z=0$.

Comme le montre la figure 22 , la réflectivité du miroir non-linéaire croît rapidement avec le gain laser. Des réflectivités supérieures à $100 \%$ sont obtenues pour un produit $\alpha_{0} L$ 
de l'amplificateur supérieur à $\sim 4$. On voit donc tout l'intérêt de cette interaction nonlinéaire qui permet d'obtenir de fortes réflectivités dues à l'amplification laser de toutes les ondes mises en jeu. Le mélange à quatre ondes a été validé expérimentalement avec des impulsions nanosecondes dans des amplificateurs $\mathrm{Nd}$ :YAG pompés par des lampes flashs, des amplificateurs $\mathrm{Nd}_{\mathrm{YVO}} \mathrm{YV}_{4}$ compacts pompés par diodes lasers et des amplificateurs Ti:Saphir $[50,51,53,54]$. Dans tous ces matériaux, des réflectivités de conjugaison de phase supérieures à $100 \%$ ont été démontrées.

\subsection{Sources lasers auto-compensées de leurs distorsions}

L'apport des deux faisceaux pompes constitue une limitation à l'utilisation du mélange à quatre ondes pour réaliser un miroir à conjugaison de phase. La conjugaison de phase auto-pompée permet quant à elle de réaliser le mélange à quatre ondes en injectant dans le milieu non-linéaire un faisceau unique. Ce faisceau traverse une première fois le milieu non-linéaire, puis y est réinjecté à l'aide d'une boucle de rétro-réaction. Cette boucle est réalisée au moyen de miroirs plans. Le faisceau ainsi renvoyé et le faisceau initial inscrivent un hologramme dans le milieu non-linéaire. Les autres faisceaux nécessaires au mélange à quatre ondes sont engendrés à partir du bruit, c'est à dire de l'émission laser spontanée. C.e type de miroir non-linéaire fonctionne si la condition

$$
\eta G p>1
$$

est vérifiée, où $\eta$ est l'efficacité de diffraction de l'hologramme inscrit, $G$ le gain présent dans la boucle et $p$, les pertes. Le gain $G$ peut provenir d'un amplificateur laser introduit dans la boucle $[55,56]$.

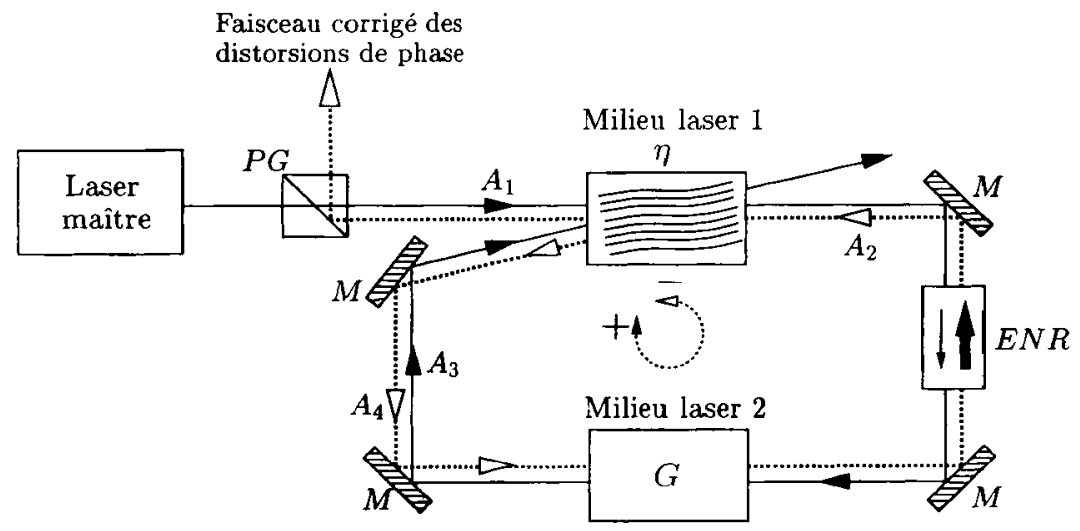

Figure 23: Schéma d'architecture laser utilisant la conjugaison de phase auto-pompée dans le milieu laser en configuration injectée. $P G$ : polariseur de Glan; $M$ : miroir plan; $E N R$ : élément optique non-réciproque (utilisant, par exemple, un rotateur de Faraday).

Cette technique a été utilisée avec succès dans le milieu laser lui-même pour réaliser des architectures lasers auto-corrigées de leurs distorsions. Ces expériences ont été faites dans des lasers à gaz [57, 58] et dans des lasers Nd:YAG [59-63]. Le principe de ces expériences est présenté sur la Figure 23. Dans la configuration injectée, l'impulsion pompe 
$A_{1}$ est délivrée par un laser maître de grande qualité de faisceau. Le faisceau $A_{1}$ traverse une première fois le milieu laser 1 , puis y est réinjecté à l'aide de la boucle de rétroréaction formée de quatre miroirs plans. Le faisceau ainsi renvoyé dans le milieu laser 1, constitue le faisceau signal $A_{3} . A_{1}$ et $A_{3}$ inscrivent alors un hologramme dynamique de gain dans le milieu laser 1 . Le faisceau pompe $A_{2}$ est engendré à partir de l'émission laser spontanée. On est donc de nouveau dans les conditions de la conjugaison de phase par mélange à quatre ondes. L'onde conjuguée $A_{4}$ est alors obtenue, par diffraction de $A_{2}$ sur l'hologramme. Après un passage dans la boucle, $A_{4}$ redevient le faisceau $A_{2}$. On voit immédiatement que la condition d'oscillation est conditionnée par le renforcement du faisceau $A_{2}$ à chaque tour. Si $n$ est le nombre de tours dans la cavité, la condition d'oscillation s'écrit: $A_{2}(n+1)>A_{2}(n)$. Comme $A_{2}(n+1)=G \times p \times A_{4}(n)$ et que $A_{4}(n)=\eta A_{2}(n)$, on retrouve la conditon $\eta G p>1$. Le gain $G$ est fourni par le milieu laser 2. Notons toutefois qu'il est en théorie possible de s'affranchir du milieu laser 2, si le milieu laser 1 possède un gain suffisant pour vérifier $\eta>1$. Pour optimiser l'efficacité de diffraction, $\eta$, de l'hologramme de gain, le rapport en énergie entre les faisceaux $A_{1}$ et $A_{3}$ doit être optimisé [64]. Pour cela, il est nécessaire d'introduire dans la boucle un élément non-réciproque qui introduit des pertes $p_{+}$sur les faisceaux tournant dans le sens " + " et si possible aucune perte dans le sens "-" $\left(p_{-}=0\right)$. Cet élément non-réciproque peut être formé d'un rotateur de Faraday et d'une lame demi-onde entourés par deux polariseurs [65]. Cet élément non-réciproque va également forcer l'oscillation dans le sens de l'onde conjuguée en phase (sens "-.") et éviter une trop forte saturation du milieu laser 2 lors du processus d'écriture de l'hologramme de gain. Comme l'hologramme inscrit dans le milieu laser 1 rend compte de toutes les distorsions de phase introduites dans la boucle, y compris celles introduites par le milieu laser servant au mélange d'ondes, I'onde conjuguée extraite possède toutes les qualités spatiales de l'onde incidente.

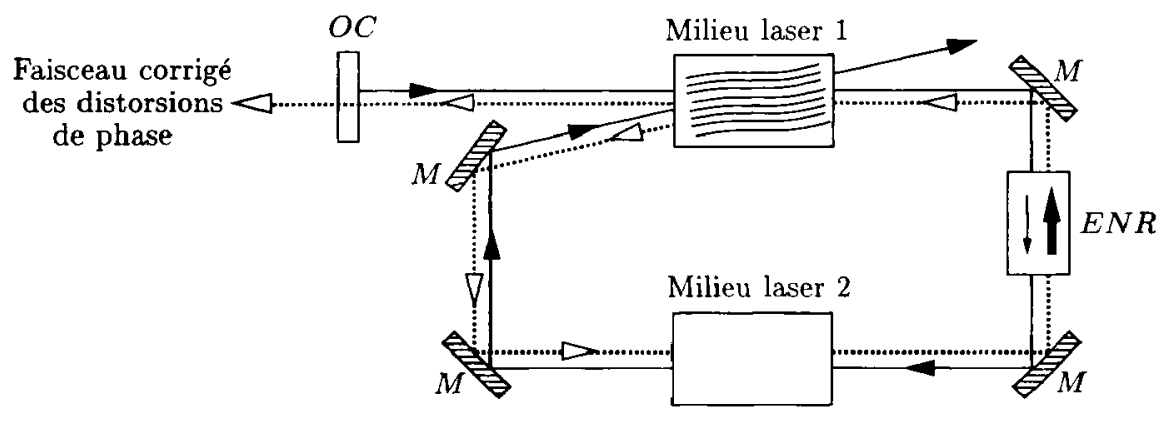

Figure 24: Schéma d'architecture laser utilisant la conjugaison de phase auto-pompée dans le milieu laser en configuration auto-oscillante. $P G$ : polariseur de Glan; $M$ : miroir plan; $E N R$ : élément optique non-réciproque; $O C$ : optique de couplage.

L'onde extraite d'une telle cavité étant entre 10 et 100 fois plus énergétique que l'onde incidente, on réalise une version auto-oscillante de cette architecture en remplaçant le laser maître par un simple miroir de couplage, $O C$, de faible coefficient de réflexion (typiquement $\sim 10 \%$ ) comme le montre la figure $24[62,66]$. Cette fois-ci toutes les ondes nécessaires au processus de mélange à quatre ondes proviennent de l'émission spontanée des amplificateurs lasers. L'onde conjuguée en phase extraite d'un tel laser possède une excellente qualité de faisceau malgré les fortes aberrations introduites par les milieux lasers. Outre la correction des distorsions de phase des amplificateurs, un tel oscillateur 


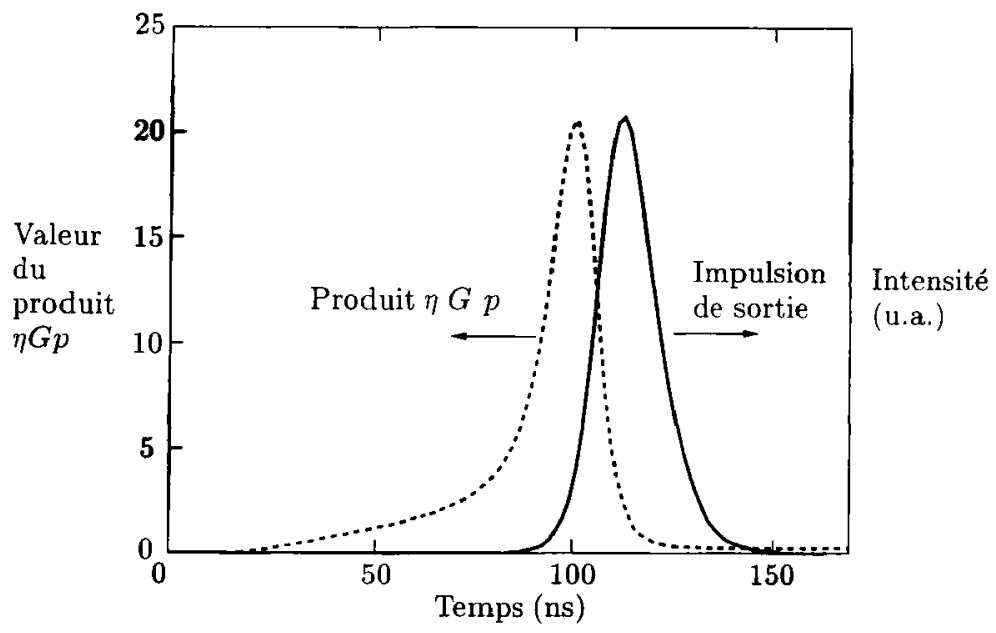

Figure 25: Dynamique de formation de l'onde conjuguée en phase dans une cavité laser à conjugaison de phase.

présente d'autres propriétés originales. Pour que l'onde puisse inscrire un hologramme, les contraintes spectrales sont telles que l'onde émise est monofréquence (monomode longitudinale). Par ailleurs, la dynamique d'écriture et d'effacement de l'hologramme par saturation du gain réalise un auto-déclenchement de la cavité. Ces propriétés sont particulièrement intéressantes puisque ce laser permet de s'affranchir d'un laser d'injection et des moyens de déclenchement usuels tels que les cellules de Pockels ou les cellules acoustooptiques. L'utilisation des équations couplées en régime transitoire permet de modéliser la dynamique d'un tel laser comme le montre la figure 25 [66]. Lorsque les deux milieux amplificateurs ont un gain suffisamment important, l'émission spontanée va commencer à inscrire un hologramme de gain dans le milieu laser 1 . Le produit $\eta G p$ va alors croître jusqu'à être supérieur à l'unité. Le processus spontané devient alors stimulé. L'onde intracavité étant de plus en plus intense, l'efficacité de l'hologramme de gain va rapidement augmenter. En revanche, lorsque l'onde qui oscille devient trop intense, elle va saturer le gain du milieu laser 2 et effacer l'holograme inscrit dans le milieu laser 1 . L'onde va alors être extraite sous la forme d'une impulsion d'une dizaine de nanoseconde.

De telles architectures ont été validées expérimentalement avec des amplificateurs Nd:YAG pompés par lampes flash ou par diodes lasers ainsi qu'avec des amplificateurs Ti:Saphir [62, 66-68].

\section{CONCLUSION}

Les effets thermiques dans les milieux lasers conduisent à des distorsions pouvant être très importantes. Ceci a pour effet de dégrader la qualité de l'onde émise ou amplifiée, d'augmenter sa divergence et donc de diminuer sa luminance. Les sources lasers qui fonctionnent à fortes énergies doivent donc inclure des éléments optiques pour corriger ces distorsions. Cette présentation donne un aperçu de quelques mécanismes non-linéaires permettant de corriger de façon adaptative et dynamique les distorsions de phase induites 
par effet thermique dans les milieux lasers.

Parmi les mécanismes non-linéaires envisageables, la diffusion Brillouin stimulée constitue une technique qui a maintenant parfaitement fait ses preuves dans des sytèmes lasers à forte puissance moyenne et à haute qualité de faisceau. L'avantage de cette technique réside dans sa simplicité d'utilisation puisqu il suffit de focaliser l'onde distordue dans une cuve contenant le milieu non-linéaire pour obtenir un faisceau conjugué en phase qui après un double passage dans le milieu aberrateur, redonne un faisceau corrigé de ses aberrations. En revanche, ces milieux sont souvent des gaz à haute pression ou des liquides toxiques, ce qui peut être une limitation à leur utilisation dans des lasers industriels.

Outre la possibilité d'obtenir un effet Brillouin dans des milieux solides, ce qui constitue une voie de recherche prometteuse encore peu étudiée, l'effet photoréfractif dans des nouveaux cristaux adaptés au proche infrarouge est une solution intéressante. Dans des régimes d'utilisation où le temps de réponse du cristal et la tenue au flux ne constituent pas un problème, l'utilisation d'un cristal photoréfractif comme miroir non-linéaire présente les avantages en terme de compacité, fidélité de conjugaison de phase, facilité d'utilisation et de manipulation.

Une solution alternative très prometteuse consiste à utiliser le milieu laser lui-même comme milieu non-linéaire pour réaliser de la conjugaison de phase. Outre la possibilité d'utiliser des matériaux lasers solides, cette technique est très efficace compte tenu de l'amplification laser subie par toutes les ondes mises en jeu. De tels miroirs non-linéaires peuvent être intégrés dans de nouvelles architectures de source laser auto-corrigées de leurs distortions de phase. Ce nouveau type de laser "tout à l'état solide" s'affranchit également des techniques usuelles de déclenchement et d'affinement spectral ce qui représente une économie de dispositifs et une souplesse d'utilisation non négligeables. Les performances déjà obtenues permettent de viser des énergies et des cadences de répétition élevées tout en maintenant une excellente qualité de faisceau quel que sòit le point de fonctionnement de la source. Les prochaines recherches sur ce sujet viseront à étendre ce mécanisme nonlinéaire à d'autres milieux lasers et d'autres longueurs d'onde. Ces techniques apparaissent bien adaptées au pompage par diodes pour la réalisation de sources hautes cadences et compactes, délivrant un faisceau proche de la limite de diffraction. En revanche, cette technique nécessite la réalisation d'amplificateurs lasers à fort gain.

Pour conclure, chaque type d'interaction non-linéaire présente des avantages et des limites. Il paraît donc difficile d'écarter une solution au profit d'une autre. Le choix d'une technique doit donc se faire en fonction du domaine d'application et des performances visées. Pour des systèmes lasers optroniques ou industriels à haute puissance moyenne o11 à forte énergie par impulsion, il est même probable que l'utilisation simultanée de plusieurs techniques non-linéaires pourra contribuer à une amélioration importante de la qualité spatiale et spectrale de ces sources.

\section{Références}

[1] Eichler H. J. , Günter P. and Pohl D. W. , Laser-induced dynamic gratings (SpringerVerlag, Berlin, 1986).

[2] Koechner W., Solid-state laser engineering (Springer-Verlag, Berlin, 1988).

[3] F. W. Quelle F. W., Appl. Opt., 5 (1966) 633-637.

[4] Foster J. D. and Osterink L. M., J. Appl. Phys., 41 (1966) 3656-3663.

[5] Eichler H. J. , Haase A., Menzel R. and Siemoneit A., J. Phys. D: Appl. Phys., 26 (1993) 1884-1891.

[6] Kaminskii A. A. and Antipenko B. M. , Multilevel Operating Schemes of Cristalline Lasers (Nauka, Moscou, 1989). 
[7] Bordé Ch. J. , "Propagation of laser beams and of atomic systems," Systèmes Fondamentaux en Optique Quantique, Les Houches, Session LIII, 1990, J. Dalibard, J. M. Raimond and J. Zinn-Justin, Eds., (Elsevier Science Pubisher, 1992) p. 288-380.

[8] Gabor D., Nature, 161 (1948) 777-778.

[9] Gabor D., Proc. R. Soc. (London) A, 197 (1949) 454-487.

[10] Zel'dovich B. Y. , Popovichev V. I. , Ragul'skii V. I. and Faizullov F. S., Sov. Phys. JETP, 15 (1972) 109-113.

[11] Nosach O. Y., Popovichev V. I., Ragul'skii V. I., and Faizullov F. S., Sov. Phys. $J E T P, 16$ (1972) 435-435.

[12] Yariv A., Appl. Phys. Lett., 28 (1976) 88.

[13] Hellwarth R. W. , J. Opt. Soc. Am., 67 (1977) 1-3.

14] Bloom D. M. and Bjorklund, Appl. Phys. Lett., 31 (1977) 592-594.

15] Yariv A. and Pepper D. M., Opt. Lett., 1 (1977) 16-18.

[16] Wang V. and Giuliano C. R., Opt. Lett., 2 (1978) 4-6.

[17] Yariv A., IEEE J. Quantum Electron., QE-14 (1978) 650-660.

[18] Fisher A., Optical Phase Conjugation (Academic press, New York, 1983).

[19] Zel'dovich B. Ya., Pilipetsky N. F. and Shkunov V. V., Principles of phase conjugation (Springer-Verlag, Berlin, 1985).

[20] Pepper D., Pour la Science, (Mars 1986) 44-54.

[21] Bordé Ch. J., "Phase conjugate optics and applications to interferometry and to laser gyros", Quantum optics, experimental gravity, and measurement theory, P. Meystre and M. O. Scully, Eds. (Plenum Publishing Corporation, 1983), p. 269-291.

[22] Rockwell D. A., IEEE J. Quant. Electron., 24 (1988) 1124-1140.

[23] Rockwell D. A., "Application of Phase Conjugation to High-Power Lasers", Optical Phase Conjugation, M. Gower and D. Proch, Eds. (Springer-Verlag, Berlin, 1994), p. $288-313$.

[24] Scott W. C. and de Wit M., Appl. Phys. Lett., 18 (1971) 3-4.

[25] Eichler H. J., Menzel R., Sander R., Schulzke M. and Schwartz J., Opt. Commun., 121 (1995) 49-54.

[26] Kaiser W. and Maier M., "Stimulated Rayleigh, Brillouin and Raman spectroscopy", Laser handbook, volume 2, F. T. Aretcchi and E. O. Schulz-Dubois, Eds. (NorthHolland Publishing Company, Amsterdam, 1972), p. 1077-1150.

[27] Dizier F., Ayral J.-L., Montel J. and Huignard J.-P., Int. J. Nonlin. Opt. Phys., 2 (1993) 229-245.

[28] Andreev N. F., Khazanov E. and Pasmanik G. A., IEEE J. Quantum Electron., 28 (1992) 330-341.

[29] Cook G. and Ridley K. D., Opt. Commun., 130 (1996) 192-204.

[30] St. Pierre J. R., Injeyan H., Hilyard R. C., Weber M. E., Berg J. G., Wickham M. G., Hoefer C. S. and Machan J. P., "One Joule per pulse, 100 Watt, diode-pumped, near diffraction limited, phase conjugated, Nd:YAG master oscillator power amplifier", OSA Proc. on Advanced Solid-State Lasers, volume 15, A. A. Pinto and T. Y. Fan, Eds. (Optical Society of America, Washington DC, 1993) p. 2-8.

[31] Dehn A., Eichler H. J., Haase A., Liu B. and Mehl O., SPIE, 2986 (1997) 74-83.

[32] Ayral J.-L., Montel J., Verny T. and Huignard J.-P., Opt. Lett., 16 (1991) 1225-1227.

[33] Eichler H. J., Haase A., Kunde J., Liu B. and Mehl O., SPIE, 2986 (1997) 46-54.

[34] Cronin-Golomb M., Fisher B., White J. O. and Yariv A., Appl. Phys. Lett., 42 (1983) 919-921.

(35) Cronin-Golomb M., Palaski J. and Yariv A., Appl. Phys. Lett., 47 (1985) 1131-1133.

[36] Korol'kov S. A., Kuzminov Y. S., Mamaev A. V., Shkunov V. V. and Zozulya A. A., J. Opt. Soc. Am. B, 9 (1992) 664-671.

[37] Mager L., Laquarnoy C., Pauliat G., Garrett M. H., Rytz D. and Roosen G., Opt. Lett., 19 (1994) 1508-1510. 
[38] Feinberg J., Opt. Lett. 7 (1982) 486-588.

[39] MacDonald K. R. and Feinberg J., J. Opt. Soc. Am., 73 (1983) 548-553.

[40] Wechsler B. A., Klein M. B., Nelson C. C. and Schwartz R. N., Opt. Lett., 19 (1994) $536-538$.

[41] Brignon A., Geffroy D., Huignard J.-P., Garrett M. H. and Mnushkina I., Opt. Commun. 137 (1997) 311-316.

[42] Huot N., Jonathan J. M. C., Rytz D. and Roosen G., Opt. Commun., 140 (1997) $296-298$.

[43] Brignon A., Huignard J.-P., Garrett M. H. and Mnushkina I., Opt. Lett., 22 (1997) $442-444$.

[44] Brignon A., Sénac S., Ayral J.-L. and Huignard J.-P., Appl. Opt., 37 (1998) 39903995.

[45] Chiou A. E. and Yeh P., Opt. Lett., 10 (1985) 621-623.

[46] Brignon A., Huignard J.-P., Garrett M. H. and Mnushkina I., Appl. Opt., 22 (1997) $442-444$.

[47] Abrams R. L. and Lind R. C., Opt. Lett., 2 (1978) 94 96, 3 (1978) 205.

[48] Fisher R. A. and Feldman B. J., Opt. Lett., 4 (1979) 140-142.

[49] Tomita A., Appl. Phys. Lett., 34 (1979) 463-464.

[50] Crofts G. J., Green R. P. M. and Damzen M. J., Opt. Lett., 17 (1992) 920-922.

[51] Brignon A. and Huignard J.-P., IEEE J. Quant. Electron., 30 (1994) 2203 2210.

(52) Siegman A.E., Lasers (University Science Books, Mill Valley, California, 1986).

[53] Brignon A., Feugnet G., Huignard J.-P. and Pocholle J.-P., Opt. Lett., 20 (1995) $548-550$.

[54] Crofts G. J., Banti X. and Damzen M. J., Opt. Lett., 20 (1995) 1634-1636.

55] Betin A. A. and Mitropol'skiř O. V., Sov. J. Quantum Electron., 17 (1987) 636-640.

[56] Odoulov S., Soskin M. and Khizniak A., Optical oscillators with degenerate four-wave mixing (dynamic grating lasers) (Harwood academic publishers, Chur, Switzerland, 1991).

[57] Bel'dyugin I. M., Zolotarev M. V., Kireev S. E. and Odintsov A. I., Sov. J. Quant. Electron., 16 (1986) 535-537.

[58] Ouhayoun M. and Guern Y., IEEE J. Quantum Electron., QE-22 (1986) 2150-2153.

[59] Bel'dyugin I. M., Berenberg V. A., Vasil'ev A. E., Mochalov I. V., Petnikova V. M., Petrovskii G. T., Kharchenko M. A. and Shuvalov V. V., Sov. J. Quantum Electron., 19 (1989) 740-742.

[60] Green R. P. M., Crofts G. J. and Damzen M. J., Opt. Lett., 19 (1994) 393-395.

61) Antipov O. L., Belyaev S. I. and Kuzhelev A. S., JETP Lett., 60 (1994) 165-170.

$62]$ Damzen M. J., Green R. P. M. and Syed K. S., Opt. Lett., 20 (1995) 1704-1706.

63) Sillard P., Brignon A. and Huignard J.-P., J. Opt. Soc. Am. B., 14 (1997) 2049-2058.

[64] Brignon A. and Huignard J.-P., Journal of Imaging Science and Technology, 41 (1997) 474-481.

[65] Damzen M. J., Green R. P. M. and Crofts G. J., Opt. Lett., 19 (1994) 34-36.

[66] Sillard P., Brignon A. and Huignard J.-P., IEEE J. Quant. Electron., 34 (1998) 465472.

[67] Sillard P., Brignon A., Huignard .J.-P. and Pocholle .J. P., Opt. Lett., 23 (1998) 10931095 .

[68] Minassian A., Crofts G. J. and Damzen M. J., Opt. Lett., 22 (1997) 697-699. 